\title{
A FEASIBLE TRUST-REGION SEQUENTIAL QUADRATIC PROGRAMMING ALGORITHM
}

\author{
STEPHEN J. WRIGHT* AND MATTHEW J. TENNY ${ }^{\dagger}$
}

\begin{abstract}
An algorithm for smooth nonlinear constrained optimization problems is described, in which a sequence of feasible iterates is generated by solving a trust-region sequential quadratic programming (SQP) subproblem at each iteration, and perturbing the resulting step to retain feasibility of each iterate. By retaining feasibility, the algorithm avoids several complications of other trust-region SQP approaches: The objective function can be used as a merit function and the SQP subproblems are feasible for all choices of the trust-region radius. Global convergence properties are analyzed under different assumptions on the approximate Hessian. Under additional assumptions, superlinear convergence to points satisfying second-order sufficient conditions is proved.
\end{abstract}

Key words. Nonlinear Constrained Optimization, Feasible Algorithm, Sequential Quadratic Programming, Trust-Region Algorithms.

AMS subject classifications. 90C30, 65K05

1. Introduction. We consider the general smooth constrained optimization problem:

$$
\min f(z) \text { subject to } c(z)=0, \quad d(z) \leq 0,
$$

where $z \in \mathrm{R}^{n}, f: \mathrm{R}^{n} \rightarrow \mathrm{R}, c: \mathrm{R}^{n} \rightarrow \mathrm{R}^{m}$, and $d: \mathrm{R}^{n} \rightarrow \mathrm{R}^{r}$ are smooth (twice continuously differentiable) functions. We denote the set of feasible points for (1.1) by $\mathcal{F}$.

At a feasible point $z$, let $H$ be an $n \times n$ symmetric matrix. The basic SQP approach obtains a step $\Delta z$ by solving the following subproblem

$$
\begin{gathered}
\min _{\Delta z} m(\Delta z) \stackrel{\text { def }}{=} \nabla f(z)^{T} \Delta z+\frac{1}{2} \Delta z^{T} H \Delta z \text { subject to } \\
c(z)+\nabla c(z)^{T} \Delta z=0, \quad d(z)+\nabla d(z)^{T} \Delta z \leq 0 .
\end{gathered}
$$

The matrix $H$ is chosen as some approximation to the Hessian of the Lagrangian, possibly obtained by a quasi-Newton technique, or possibly a "partial Hessian" computed in some application-dependent way from some of the objective and constraint functions and Lagrange multiplier estimates. The function $m(\cdot)$ is the quadratic model for the change in $f$ around the current point $z$.

Although the basic approach (1.2) often works well in the vicinity of a solution to (1.1), trust-region or line-search devices must be added to improve its robustness and global convergence behavior. In this paper, we consider a trust region of the form

$$
\|D \Delta z\|_{p} \leq \Delta
$$

where the scaling matrix $D$ is uniformly bounded above and $p \in[1, \infty]$. The choice $p=\infty$ makes (1.2), (1.3) a quadratic program, since we can then restate the trustregion constraint as $-\Delta e \leq D \Delta z \leq \Delta e$, where $e=(1,1, \ldots, 1)^{T}$. The choice $p=2$ produces the quadratic constraint $\Delta z^{T} D^{T} D \Delta z \leq \Delta^{2}$, and since $z$ is feasible for (1.1),

\footnotetext{
${ }^{*}$ Computer Sciences Department, 1210 W. Dayton Street, University of Wisconsin, Madison, WI 53706, USA; swright@cs.wisc.edu

${ }^{\dagger}$ ExxonMobil Upstream Research Company, Box 2189, Houston, TX 77252-2189, USA; tenny@bevo.che.wisc.edu
} 
we can show that the solution $\Delta z$ of (1.2), (1.3) is identical to the solution of (1.2) alone, with $H$ replaced by $H+\gamma D^{T} D$ for some $\gamma \geq 0$. For generality, we develop most of the convergence theory to apply to any choice of $p \in[1, \infty]$, making frequent use of the equivalence between $\|\cdot\|_{p}$ and $\|\cdot\|_{2}$.

By allowing $D$ to have zero eigenvalues, the constraint (1.3) generally allows $\Delta z$ to be unrestricted by the trust region in certain directions. We assume, however, that the combination of (1.3) and (1.2b) ensures that the all components of the step are controlled by the trust region; see Assumption 1 below.

When the iterate $z$ is not feasible for the original problem (1.1), we cannot in general simply add the restriction (1.3) to the constraints in the subproblem (1.2), since the resulting subproblem will be infeasible for small $\Delta$. Practical trust-region methods such as those due to Celis-Dennis-Tapia [3] and Byrd-Omojokun [12] do not insist on satisfaction of the constraints $(1.2 \mathrm{~b})$ by the step $\Delta z$, but rather achieve some reduction in the infeasibility, while staying within the trust region (1.3) and reducing the objective in the subproblem (1.2a).

Another issue that arises in the practical SQP methods is the use of a merit or penalty function to measure the worth of each point $z$. Typically this function is some combination of the objective $f(z)$ and the violations of the constraints, that is, $\left|c_{i}(z)\right|, i=1,2, \ldots, m$ and $d_{i}^{+}(z), i=1,2, \ldots, r$. The merit function may also depend on estimates of the Lagrange multipliers for the constraints in (1.1). It is sometimes difficult to choose weighting parameters in these merit functions appropriately, in a way that drives the iterates to a solution (or at least a point satisfying Karush-KuhnTucker conditions) of (1.1).

In this paper, we propose an algorithm called Algorithm FP-SQP (for feasibility perturbed SQP), in which all iterates $z^{k}$ are feasible; that is, $z^{k} \in \mathcal{F}$ for all $k$. We obtain a step by solving a problem of the form (1.2) at a feasible point $z \in \mathcal{F}$ with a trust-region constraint (1.3). We then find a perturbation $\widetilde{\Delta z}$ of the step $\Delta z$ that satisfies two crucial properties: First, feasibility:

$$
z+\widetilde{\Delta z} \in \mathcal{F}
$$

Second, asymptotic exactness: There is a continuous monotonically increasing function $\phi: \mathrm{R}^{+} \rightarrow[0,1 / 2]$ with $\phi(0)=0$ such that

$$
\|\Delta z-\widetilde{\Delta z}\|_{2} \leq \phi\left(\|\Delta z\|_{2}\right)\|\Delta z\|_{2} .
$$

Note that because $\phi(t) \leq 1 / 2$ for all $t \geq 0$, we have that

$$
(1 / 2)\|\Delta z\|_{2} \leq\|\widetilde{\Delta z}\|_{2} \leq(3 / 2)\|\Delta z\|_{2} .
$$

These conditions on $\widetilde{\Delta z}$ suffice to prove good global convergence properties for the algorithm. Additional assumptions on the feasibility perturbation technique can be made to obtain fast local convergence; see Section 4.

The effectiveness of our method depends on its being able to calculate efficiently a perturbed step $\widetilde{\Delta z}$ with the properties (1.4) and (1.5). This task is not difficult for certain structured problems, including some problems in optimal control. Additionally, in the special case in which the constraints $c$ and $d$ are linear, we can simply set $\widetilde{\Delta z}=\Delta z$. When some constraints are nonlinear, $\widetilde{\Delta z}$ can be obtained from the projection of $z+\Delta z$ onto the feasible set $\mathcal{F}$. For general problems, this projection is nontrivial to compute, but for problems with structured constraints, it may be inexpensive. 
By maintaining feasible iterates, our method gains several advantages. First, the trust region restriction (1.3) can be added to the SQP problem (1.2) without concern as to whether it will yield an infeasible subproblem. There is no need for a compositestep approach such as those mentioned above $[3,12]$. Second, the objective function $f$ can itself be used as a merit function. Third, if the algorithm is terminated early, we will be able to use the latest iterate $z^{k}$ as a feasible suboptimal point, which in many applications is far preferable to an infeasible suboptimum.

The advantages of the previous paragraph are of course shared by other feasible SQP methods. The FSQP approach described in Lawrence and Tits [10] (based on an earlier version of Panier and Tits [13] and also using ideas from Birge, Qi, and Wei [2]) calculates the main search direction via a modified SQP subproblem that includes a parameter for "tilting" the search direction toward the interior of the set defined by the inequality constraints. A second subproblem is solved to obtain a second-order correction, and an "arc search" is performed along these two directions to find a new iterate that satisfies feasibility as well as a sufficient decrease condition in the objective $f$. The approach can also handle nonlinear equality constraints, but feasibility is not maintained with respect to these constraints, in general. Our algorithm below differs in that it uses a trust region rather than arc searches to attain global convergence, it requires feasibility with respect to both inequality and equality constraints at each iteration, and it is less specific than in [10] about the details of calculating the step. In this sense, Algorithm FP-SQP represents an algorithmic framework rather than a specific algorithm.

Heinkenschloss [8] considers projected SQP methods for problems with equality constraints in addition to bounds on a subset of the variables. He specifically targets optimal control problems with bounds on the controls - a similar set of problems to those we discuss in a companion manuscript [15]. The linearized equality constraints are used to express the free variables in terms of the bounded variables, and a projected Newton direction (see [1]) is constructed for the bounded variables. The step is computed by performing a line search along this direction with projection onto the bound constraints. Besides using a line search rather than a trust region, this method contrasts with ours in that feasibility is not enforced with respect to the equality constraints, so that an augmented Lagrangian merit function must be used to determine an acceptable step length.

Other related work includes the feasible algorithm for problems with convex constraints discussed in Conn, Gould, and Toint [5]. At each iteration, this algorithm seeks an approximate minimizer of the model function over the intersection of the trust region with the original feasible set. The algorithm is targeted to problems in which the constraint set is simple (especially bound-constrained problems with $\infty$ norm trust regions, for which the intersection is defined by componentwise bounds). Aside from not requiring convexity, our method could be viewed as a particular instance of Algorithm 12.2.1 of [5, p. 452], in which the model function approximates the Lagrangian and the trial step is a perturbed SQP step. It may then be possible to apply the analysis of [5], once we show that the step generated in this fashion satisfies the assumptions in [5], at least for sufficiently small values of the trust-region radius. It appears nontrivial, however, to put our algorithm firmly into the framework of [5], and to extend the latter algorithm to handle a class of problems (featuring nonconvexity) which its designers did not have in mind. Therefore, we present an analysis that was developed independently of that in [5]. We note that several features of the analysis in [5, Chapter 12] are similar to ours; for instance, $\chi$ on [5, p. 452] is similar to 
$C(z, 1)$ defined below in (3.1), except that minimization in $\chi$ is taken over the original feasible set rather than over its linearized approximation, as in (3.1). Other aspects of the analysis in [5] and this paper are different; for instance, the generalized Cauchy point in [5, Section 12.2.1] is defined in a much more complex fashion with respect to the projected-gradient path, rather than along the straight line as in Lemma 3.3 below.

The remainder of the paper is structured as follows. The algorithm is specified in Section 2, and in Section 2.1 we show that it is possible to find a feasible perturbation of the SQP step that satisfies the requirements (1.4) and (1.5). We present global convergence results in Section 3. After some basic lemmas in Section 3.1, we describe in Section 3.2 conditions under which the algorithm has at least one limit point that either fails a constraint qualification or satisfies Karush-Kuhn-Tucker conditions. In particular, we assume in this section that the approximate Hessian $H_{k}$ in (1.2) satisfies the bound $\left\|H_{k}\right\|_{2} \leq \sigma_{0}+\sigma_{1} k$ for some constant $\sigma_{0}$ and $\sigma_{1}$-a type of bound often satisfied by quasi-Newton update formulae. In Section 3.3, we make the stronger assumption that $\left\|H_{k}\right\|$ is uniformly bounded, and prove the stronger result that all limit points of the algorithm either fail a constraint qualification or else satisfy KarushKuhn-Tucker conditions. Under stronger assumptions on the limit point $z^{*}$ and the feasibility projection technique, we prove fast local convergence in Section 4. Some final comments appear in Section 5.

A companion report of Tenny, Wright, and Rawlings [15] describes application of the algorithm to nonlinear optimization problems arising in model predictive control.

1.1. Optimality Results and Notation. The Lagrangian function for (1.1) is

$$
\mathcal{L}(z, \mu, \lambda) \stackrel{\text { def }}{=} f(z)+\mu^{T} c(z)+\lambda^{T} d(z),
$$

where $\mu \in \mathrm{R}^{m}$ and $\lambda \in \mathrm{R}^{r}$ are Lagrange multipliers for the constraints. The KarushKuhn-Tucker (KKT) conditions for (1.1) are as follows:

$$
\begin{aligned}
\nabla_{z} \mathcal{L}(z, \mu, \lambda)=\nabla f(z)+\nabla c(z) \mu+\nabla d(z) \lambda & =0, \\
c(z) & =0, \\
0 \geq d(z) & \perp \lambda \geq 0,
\end{aligned}
$$

where $\perp$ indicates that $\lambda^{T} d(z)=0$. We refer to any point $z$ such that there exist $\mu$ and $\lambda$ satisfying the conditions (1.8) as a KKT point.

For any feasible point $z$, we denote the active set $\mathcal{A}(z)$ as follows:

$$
\mathcal{A}(z) \stackrel{\text { def }}{=}\left\{i=1,2, \ldots, r \mid d_{i}(z)=0\right\} .
$$

To ensure that the tangent cone to the constraint set at a feasible point $z$ adequately captures the geometry of the feasible set near $z$, a constraint qualification must be satisfied at $z$. In the global convergence analysis of Section 3, we use the Mangasarian-Fromovitz constraint qualification (MFCQ), which requires that

$$
\begin{gathered}
\nabla c(z) \text { has full column rank; and } \\
\text { there exists a vector } v \in \mathrm{R}^{n} \text { such that } \\
\nabla c(z)^{T} v=0 \text { and } v^{T} \nabla d_{i}(z)<0 \text { for all } i \in \mathcal{A}(z) .
\end{gathered}
$$

A more stringent constraint qualification, used in the local convergence analysis of Section 4 , is the linear independence constraint qualification (LICQ), which requires 
that

$\left\{\nabla c_{i}(z), i=1,2, \ldots, m\right\} \cup\left\{\nabla d_{i}(z), i \in \mathcal{A}(z)\right\}$ is linearly independent.

If $z$ is a solution of (1.1), at which a constraint qualification such as (1.10) or (1.11) is satisfied, there exist vectors $\mu$ and $\lambda$ such that (1.8) is satisfied by the triplet $(z, \mu, \lambda)$.

We say that the strict complementarity condition is satisfied at the KKT point $z$ if for some choice of the Lagrange multiplier vectors $\mu$ and $\lambda$ satisfying the conditions (1.8), we have

$$
\lambda-d(z)>0
$$

That is, $\lambda_{i}>0$ for all $i \in \mathcal{A}(z)$.

We use $\mathcal{B}(z, t)$ to denote the open ball (in the Euclidean norm) of radius $t$ about $z$. When the subscript on the norm $\|\cdot\|$ is omitted, the Euclidean norm $\|\cdot\|_{2}$ is to be understood. The closure of a set $L$ is denoted by $\operatorname{cl}(L)$.

We use order notation in the following way: If two matrix, vector, or scalar quantities $M$ and $A$ are functions of a common quantity, we write $M=O(\|A\|)$ if there is a constant $\beta$ such that $\|M\| \leq \beta\|A\|$ whenever $\|A\|$ is sufficiently small. We write $M=\Omega(\|A\|)$ if there is a constant $\beta$ such that $\|M\| \geq \beta^{-1}\|A\|$ whenever $\|A\|$ is sufficiently small. We write $M=o(\|A\|)$ if there is a continuous, increasing function $\phi: \mathrm{R} \rightarrow \mathrm{R}$ with $\phi(0)=0$ such that $\|M\| \leq \phi(\|A\|)\|A\|$ for all $\|A\|$ sufficiently small.

2. The Algorithm. In specifying the algorithm, we assume only that the perturbed step $\widetilde{\Delta z}$ satisfies (1.4) and (1.5), without specifying how it is calculated. As with all trust-region algorithms, a critical role is played by the ratio of actual to predicted decrease, which is defined for a given $\mathrm{SQP}$ step $\Delta z^{k}$ and its perturbed counterpart $\widetilde{\Delta z}^{k}$ as follows:

$$
\rho_{k}=\frac{f\left(z^{k}\right)-f\left(z^{k}+\widetilde{\Delta z}^{k}\right)}{-m_{k}\left(\Delta z^{k}\right)} .
$$

The algorithm is specified as follows.

Algorithm 2.1 (FP-SQP).

Given starting point $z_{0} \in \mathcal{F}$, initial radius $\Delta_{0} \in(0, \bar{\Delta}]$, initial scaling matrix $D_{0}$, trust-region upper bound $\bar{\Delta} \geq 1, \eta \in(0,1 / 4)$, and $p \in[1, \infty]$;

for $k=0,1,2, \cdots$

Obtain $\Delta z^{k}$ by solving (1.2), (1.3);

if $m_{k}\left(\Delta z^{k}\right)=0$

STOP;

Seek $\widetilde{\Delta z}^{k}$ with the properties (1.4) and (1.5);

if no such $\widetilde{\Delta z}^{k}$ is found;

$$
\begin{aligned}
& \Delta_{k+1} \leftarrow(1 / 2)\left\|D_{k} \Delta z^{k}\right\|_{p} ; \\
& z^{k+1} \leftarrow z^{k} ; D_{k+1} \leftarrow D_{k} ;
\end{aligned}
$$

else

Calculate $\rho_{k}$ using (2.1);

if $\rho_{k}<1 / 4$

$\Delta_{k+1} \leftarrow(1 / 2)\left\|D_{k} \Delta z^{k}\right\|_{p} ;$

else if $\rho_{k}>3 / 4$ and $\left\|D_{k} \Delta z^{k}\right\|_{p}=\Delta_{k}$ 
end (for).

$$
\begin{gathered}
\text { else } \begin{array}{c}
\Delta_{k+1} \leftarrow \min \left(2 \Delta_{k}, \bar{\Delta}\right) ; \\
\Delta_{k+1} \leftarrow \Delta_{k} ;
\end{array} \\
\text { if } \rho_{k} \geq \eta \\
z^{k+1} \leftarrow z^{k}+\widetilde{\Delta z}^{k} ; \\
\text { choose new scaling matrix } D_{k+1} ;
\end{gathered}
$$

else

$$
z^{k+1} \leftarrow z^{k} ; D_{k+1} \leftarrow D_{k}
$$

We now state some assumptions that are used in the subsequent analysis. First, we define the level set $L_{0}$ as follows:

$$
L_{0} \stackrel{\text { def }}{=}\left\{z \mid c(z)=0, d(z) \leq 0, f(z) \leq f\left(z_{0}\right)\right\} \subset \mathcal{F} .
$$

Our assumption on the trust-region bound (1.3) is as follows:

Assumption 1. There is a constant $\delta$ such that for all points $z \in L_{0}$ and all scaling matrices $D$ used by the algorithm, the following conditions hold:

(a) $D$ is uniformly bounded; and

(b) we have for any $\Delta z$ satisfying the constraints

$$
c(z)+\nabla c(z)^{T} \Delta z=0, \quad d(z)+\nabla d(z)^{T} \Delta z \leq 0
$$

that

$$
\delta^{-1}\|\Delta z\|_{2} \leq\|D \Delta z\|_{p} \leq \delta\|\Delta z\|_{2} .
$$

In this assumption, the constant that relates $\|\cdot\|_{2}$ with the equivalent norms $\|\cdot\|_{p}$ for all $p$ between 1 and $\infty$ is absorbed into $\delta$. Note that the right-hand inequality in (2.2) is implied by part (a) of the assumption.

Note that for unconstrained problems (in which $c$ and $d$ are vacuous), the lefthand inequality in (2.2) is satisfied when all scaling matrices $D$ used by the algorithm have bounded inverse. Another special case of relevance to optimal control problems occurs when the constraints have the form

$$
c(u, v)=0, \quad c: \mathrm{R}^{n-m} \times \mathrm{R}^{m} \rightarrow \mathrm{R}^{m},
$$

(that is $u \in \mathrm{R}^{n-m}$ and $v \in \mathrm{R}^{m}$ ), and the trust-region constraint is imposed only on the $u$ variables; that is,

$$
\left\|D_{u} \Delta u\right\|_{p} \leq \Delta
$$

where $D_{u}$ is a diagonal matrix with positive diagonal elements. The linearized constraints $(1.2 \mathrm{~b})$ then have the form

$$
\nabla_{u} c(u, v)^{T} \Delta u+\nabla_{v} c(u, v)^{T} \Delta v=0
$$

which if $\nabla_{v} c(u, v)$ is invertible leads to

$$
\Delta v=-\left(\nabla_{v} c(u, v)\right)^{-T} \nabla_{u} c(u, v)^{T} \Delta u .
$$

If we assume that $\nabla_{v} c(u, v)$ is invertible for all points $(u, v)$ in the region of interest, with $\left\|\left(\nabla_{v} c(u, v)\right)^{-1}\right\|$ bounded, we can define a constant $\hat{\delta}>0$ such that $\|\Delta v\|_{p} \leq$ $\hat{\delta}\|\Delta u\|_{p}$. We then have

$$
\|(\Delta u, \Delta v)\|_{p} \leq(1+\hat{\delta})\|\Delta u\|_{p} \leq(1+\hat{\delta}) D_{\min }^{-1}\left\|D_{u} \Delta u\right\|_{p}=(1+\hat{\delta}) D_{\min }^{-1}\|D(\Delta u, \Delta v)\|_{p},
$$


where we define $D_{\min }$ to be a lower bound on the diagonals of $D_{u}$, and $D=\operatorname{diag}\left(D_{u}, 0\right)$. On the other hand, we have

$$
\|D(\Delta u, \Delta v)\|_{p}=\left\|D_{u} \Delta u\right\|_{\infty} \leq D_{\max }\|\Delta u\|_{p} \leq D_{\max }\|(\Delta u, \Delta v)\|_{p},
$$

where $D_{\max }$ is an upper bound on the diagonals of $D_{u}$. It follows from the last two expressions that Assumption 1 is satisfied in this situation.

Second, for some results we make an assumption on the boundedness of the level set $L_{0}$ and on the smoothness of

Assumption 2. The level set $L_{0}$ is bounded, and the functions $f$, $c$, and $d$ in (1.1) are twice continuously differentiable in an open neighborhood $\mathcal{N}\left(L_{0}\right)$ of this set.

Note that $L_{0}$ is certainly closed, so that if Assumption 2 holds, it is also compact.

2.1. Algorithm FP-SQP is Well Defined. We show first that the algorithm is well defined, in the sense that given a feasible point $z_{k}$, a step $\widetilde{\Delta z_{k}}$ satisfying (1.4) and (1.5) can be found for all sufficiently small $\Delta_{k}$, under certain assumptions.

We note first that whenever $z=z_{k}$ is feasible and Assumption 1 holds, the subproblem (1.2), (1.3) has a solution. This fact follows from nonemptiness, closedness, and boundedness of the feasible set for the subproblem. To show that there exists $\widetilde{\Delta z_{k}}$ satisfying (1.4) and (1.5), we make use of the following assumption.

Assumption 3. For every point $\hat{z} \in L_{0}$, there are positive quantities $\zeta$ and $\hat{\Delta}_{3}$ such that for all $z \in \operatorname{cl}\left(\mathcal{B}\left(\hat{z}, \delta \hat{\Delta}_{3}\right)\right)$ we have

$$
\min _{v \in \mathcal{F}}\|v-z\| \leq \zeta\left(\|c(z)\|+\left\|[d(z)]_{+}\right\|\right),
$$

where $\delta$ is the constant from Assumption 1 and $[d(z)]_{+}=\left[\max \left(d_{i}(z), 0\right)\right]_{i=1}^{r}$.

(Recall our convention that $\|\cdot\|$ denotes $\|\cdot\|_{2}$.)

This assumption requires the constraint system to be regular enough near each feasible point that a bound like that of Hoffmann [9] for systems of linear equalities and inequalities is satisfied. Assumption 3 is essentially the same as Assumption C of Lucidi, Sciandrone, and Tseng [11]. A result of Robinson [14, Corollary 1] shows that Assumption 3 is satisfied whenever MFCQ is satisfied at all points in $L_{0}$. The following result shows that a bound similar to (2.6) also holds locally, in the vicinity of a feasible point satisfying MFCQ.

LEMMA 2.1. Let $\hat{z}$ be a feasible point for (1.1) at which $M F C Q$ is satisfied. Then there exist positive quantities $\zeta$ and $\hat{R}_{1}$ such that for all $z \in \operatorname{cl}\left(\mathcal{B}\left(\hat{z}, \hat{R}_{1}\right)\right)$, the bound (2.6) is satisfied.

Proof. We first choose $\bar{R}_{1}$ small enough that for all $\tilde{z} \in \operatorname{cl}\left(\mathcal{B}\left(\hat{z}, \bar{R}_{1}\right)\right) \cap \mathcal{F}$, we have that $\mathcal{A}(\tilde{z}) \subset \mathcal{A}(\hat{z})$, where $\mathcal{A}(\cdot)$ is defined by (1.9). Let $v$ be a vector satisfying (1.10) at $z=\hat{z}$, and assume without loss of generality that $\|v\|_{2}=1$. Because $\nabla c(\hat{z})$ has full column rank, we have by decreasing $\bar{R}_{1}$ if necessary that for any $\tilde{z} \in \operatorname{cl}\left(\mathcal{B}\left(\hat{z}, \bar{R}_{1}\right)\right)$, $\nabla c(\tilde{z})$ also has full column rank. Moreover, using the full rank of $\nabla c(\tilde{z})$, we can find a perturbation $\tilde{v}$ of $v$ satisfying $\|\tilde{v}-v\|=O(\|\tilde{z}-\hat{z}\|)$ and (after possibly decreasing $\bar{R}_{1}$ again) $\|\tilde{v}\| \geq 0.5$, such that

$$
\nabla c(\tilde{z})^{T} \tilde{v}=0 \text { and } \tilde{v}^{T} \nabla d_{i}(\tilde{z})<0 \text { for all } i \in \mathcal{A}(\hat{z}) \supset \mathcal{A}(\tilde{z}) \text {, all } \tilde{z} \in \operatorname{cl}\left(\mathcal{B}\left(\hat{z}, \bar{R}_{1}\right)\right) \cap \mathcal{F} \text {. }
$$

Hence, the MFCQ condition is satisfied for all $\tilde{z} \in \operatorname{cl}\left(\mathcal{B}\left(\hat{z}, \bar{R}_{1}\right)\right) \cap \mathcal{F}$.

We now appeal to Corollary 1 of Robinson [14]. From this result, we have that there is $\zeta>0$ (depending on $\hat{z}$ but not on $\tilde{z}$ ) and an open neighborhood $M(\tilde{z})$ of each $\tilde{z} \in \operatorname{cl}\left(\mathcal{B}\left(\hat{z}, \bar{R}_{1}\right)\right) \cap \mathcal{F}$ such that (2.6) holds for all $z \in M(\tilde{z})$. Since

$$
\hat{M}(\hat{z}) \stackrel{\text { def }}{=} \cup_{\tilde{z}}\left\{M(\tilde{z}) \mid \tilde{z} \in \operatorname{cl}\left(\mathcal{B}\left(\hat{z}, \bar{R}_{1}\right)\right) \cap \mathcal{F}\right\}
$$


is an open neighborhood of the compact $\operatorname{set} \operatorname{cl}\left(\mathcal{B}\left(\hat{z}, \bar{R}_{1}\right)\right) \cap \mathcal{F}$, we can define $\hat{R}_{1} \leq \bar{R}_{1}$ small enough that $\operatorname{cl}\left(\mathcal{B}\left(\hat{z}, \hat{R}_{1}\right)\right) \subset \hat{M}(\hat{z})$. Thus, since (2.6) holds for all $z \in M(\tilde{z})$, our proof is complete.

We observed above that under Assumption 1, the solution $\Delta z$ of (1.2), (1.3) is well defined. Using the other assumptions, we now show that $\widetilde{\Delta z}$ satisfying the properties (1.4) and (1.5) can also be found, so that Algorithms FP-SQP is well defined.

THEOREM 2.2. Suppose that Assumptions 1, 2, and 3 are satisfied. Then there is a positive constant $\Delta_{\text {def }}$ such that for any $z \in L_{0}$ and any $\Delta \leq \Delta_{\text {def }}$, there is a step $\widetilde{\Delta z}$ that satisfies the properties (1.4) and (1.5), where $\Delta z$ is the solution of (1.2), (1.3) for the given values of $z$ and $\delta$.

Proof. We show that the result holds for the function $\phi(t)=\min (1 / 2, \sqrt{t})$ in (1.5).

We first choose $\hat{\Delta}_{0}$ small enough that $\mathcal{B}\left(z, \delta \hat{\Delta}_{0}\right) \subset \mathcal{N}\left(L_{0}\right)$ for all $z \in L_{0}$, where $\mathcal{N}\left(L_{0}\right)$ is defined in Assumption 2. Thus, for $\Delta \leq \hat{\Delta}_{0}$ and $\Delta z$ solving (1.2), (1.3), we have for all $\alpha \in[0,1]$ that

$$
\|\alpha \Delta z\| \leq\|\Delta z\| \leq \delta\|D \Delta z\|_{p} \leq \delta \hat{\Delta}_{0}
$$

so that $z+\alpha \Delta z \in \mathcal{N}\left(L_{0}\right)$.

Given any $\hat{z} \in L_{0}$, we seek a positive constant $\hat{\Delta}$ such that for all $z \in \operatorname{cl}(\mathcal{B}(\hat{z}, \delta \hat{\Delta} / 2)) \cap \mathcal{F}$, and all $\Delta \leq \hat{\Delta} / 2$, there is a step $\widetilde{\Delta z}$ that satisfies the properties (1.4) and (1.5).

We choose initially $\hat{\Delta}=\hat{\Delta}_{0}$, and assume that $\Delta z$ satisfies $\|D \Delta z\|_{p} \leq \Delta$, which implies from Assumption 1 and the definitions of $\Delta$ and $\hat{\Delta}$ that

$$
\|\Delta z\| \leq \delta\|D \Delta z\|_{p} \leq \delta \Delta \leq \delta \hat{\Delta} / 2<\delta \hat{\Delta}_{0} .
$$

From feasibility of $z,(2.7)$, and (1.2b), and the fact that $c$ and $d$ are twice continuously differentiable in $\mathcal{N}\left(L_{0}\right)$, we have that

$$
c(z+\Delta z)=c(z)+\nabla c(z)^{T} \Delta z+O\left(\|\Delta z\|^{2}\right)=O\left(\|\Delta z\|^{2}\right)
$$

and

$$
[d(z+\Delta z)]_{+}=\left[d(z)+\nabla d(z)^{T} \Delta z+O\left(\|\Delta z\|^{2}\right)\right]_{+}=O\left(\|\Delta z\|^{2}\right) .
$$

We now set $\hat{\Delta} \leftarrow \min \left(\hat{\Delta}, \hat{\Delta}_{3}\right)$ and apply Assumption 3. Since

$$
\|(z+\Delta z)-\hat{z}\| \leq\|z-\hat{z}\|+\|\Delta z\| \leq \delta \hat{\Delta} / 2+\delta \hat{\Delta} / 2 \leq \delta \hat{\Delta}_{3},
$$

we have from Assumption 3 and the estimates above that

$$
\min _{v \in \mathcal{F}}\|v-(z+\Delta z)\| \leq \zeta\left(\|c(z+\Delta z)\|+\left\|[d(z+\Delta z)]_{+}\right\|\right)=O\left(\zeta\|\Delta z\|^{2}\right),
$$

where $\zeta$ may depend on $\hat{z}$. Since $v=z$ is feasible for (2.8), we have that any solution of this projection problem satisfies $\|v-(z+\Delta z)\| \leq\|\Delta z\|$. Hence, the minimization on the left-hand side of (2.8) may be restricted to the nonempty compact $\operatorname{set} \operatorname{cl}(\mathcal{B}(z+$ $\Delta z,\|\Delta z\|)) \cap \mathcal{F}$, so the minimum is attained. If we use the minimizer $v$ to define $\widetilde{\Delta z}=v-z$, then from (2.8) we have

$$
\|\widetilde{\Delta z}-\Delta z\|=O\left(\zeta\|\Delta z\|^{2}\right) .
$$


Therefore, by decreasing $\hat{\Delta}$ if necessary, we find that (1.5) is satisfied for our choice $\phi(t)=\min (1 / 2, \sqrt{t})$.

The set of open Euclidean balls $\mathcal{B}(\hat{z}, \delta \hat{\Delta} / 2), \hat{z} \in L_{0}$ forms an open cover of $L_{0}$. Since $L_{0}$ is compact, we can define a finite subcover. By defining $\Delta_{\text {def }}$ to be the minimum of the $\hat{\Delta} / 2$ over the subcover, we have that $\Delta_{\text {def }}$ is positive and has the desired property.

3. Global Convergence. In this section, we prove convergence to KKT points of (1.1). Our results are of two types. We show first in Section 3.2 that if Algorithm FP-SQP does not terminate finitely (at a KKT point), it has a limit point which either satisfies the MFCQ and KKT conditions or else fails to satisfy MFCQ. In Section 3.3, we show under a stronger assumption on the approximate Hessian $H_{k}$ that all limit points either fail to satisfy MFCQ or else satisfy both MFCQ and KKT.

We start with some technical results.

3.1. Technical Results. The first result concerns the solution of a linear programming variant of the SQP subproblem (1.2), (1.3). Its proof appears in the Appendix.

Lemma 3.1. Let $f, c$, and $d$ be as defined in (1.1), and let $C(z, \tau)$ denote the negative of the value function of the following problem, for some $z \in \mathcal{F}$ and $\tau>0$ :

$$
\begin{aligned}
\operatorname{CLP}(z, \tau): & \min _{w} \nabla f(z)^{T} w \text { subject to } \\
c(z)+\nabla c(z)^{T} w & =0, \quad d(z)+\nabla d(z)^{T} w \leq 0, \quad w^{T} w \leq \tau^{2} .
\end{aligned}
$$

For any point $\bar{z} \in \mathcal{F}$, we have $C(\bar{z}, 1) \geq 0$, with $C(\bar{z}, 1)=0$ if and only if $\bar{z}$ is a KKT point (1.8).

When the MFCQ conditions (1.10) are satisfied at $\bar{z}$, but $\bar{z}$ is not a KKT point, then there exist positive quantities $R_{2}$ and $\epsilon$ such that for any $z \in \mathcal{B}\left(\bar{z}, R_{2}\right) \cap \mathcal{F}$, we have $C(z, 1) \geq \epsilon$.

An immediate consequence of this result is that for any subsequence $\left\{z^{k}\right\}_{k \in \mathcal{K}}$ such that $z^{k} \rightarrow \bar{z}$ and $C\left(z^{k}, 1\right) \rightarrow 0$, where $\bar{z}$ satisfies the MFCQ conditions, we must have that $\bar{z}$ is a KKT point for (1.1).

Note that $C(z, \tau)$ is an increasing concave function of $\tau>0$. In particular, if $w(z, \tau)$ attains the optimum in $\operatorname{CLP}(z, \tau)$, the point $\alpha w(z, \tau)$ is feasible in $\operatorname{CLP}(z, \alpha \tau)$ for all $\alpha \in[0,1]$, so that

$$
C(z, \alpha \tau) \geq \alpha C(z, \tau), \quad \text { for all } \tau>0, \text { all } \alpha \in[0,1] .
$$

For convenience, we restate the subproblem (1.2), (1.3) at an arbitrary feasible point $z$ as follows:

$$
\begin{gathered}
\min _{\Delta z} m(\Delta z) \stackrel{\text { def }}{=} \nabla f(z)^{T} \Delta z+\frac{1}{2} \Delta z^{T} H \Delta z \quad \text { subject to } \\
c(z)+\nabla c(z)^{T} \Delta z=0, \quad d(z)+\nabla d(z)^{T} \Delta z \leq 0 \\
\|D \Delta z\|_{p} \leq \Delta
\end{gathered}
$$

where $D$ satisfies Assumption 1. Consider now the following problem, obtained by omitting the quadratic term from (3.3a):

$$
\begin{gathered}
\min _{\Delta z^{\mathrm{L}}} \nabla f(z)^{T} \Delta z^{\mathrm{L}} \quad \text { subject to } \\
c(z)+\nabla c(z)^{T} \Delta z^{\mathrm{L}}=0, \quad d(z)+\nabla d(z)^{T} \Delta z^{\mathrm{L}} \leq 0, \\
\left\|D \Delta z^{\mathrm{L}}\right\|_{p} \leq \Delta .
\end{gathered}
$$


Denote the negative of the value function for this problem by $V(z, D, \Delta)$. Referring to (3.1) and Assumption 1, we see that the feasible region for $\operatorname{CLP}\left(z, \delta^{-1} \Delta\right)$ is contained in the feasible region for (3.4), and the objectives are the same. Hence for $\Delta \in(0,1]$, we have from (3.2) that

$$
V(z, D, \Delta) \geq C\left(z, \delta^{-1} \Delta\right) \geq \delta^{-1} C(z, 1) \Delta .
$$

For $\Delta>1$, on the other hand, we have

$$
V(z, D, \Delta) \geq C\left(z, \delta^{-1} \Delta\right) \geq \delta^{-1} C(z, \Delta) \geq \delta^{-1} C(z, 1) .
$$

Hence, by combining these observations, we obtain that

$$
V(z, D, \Delta) \geq \delta^{-1} C(z, 1) \min (1, \Delta) .
$$

The following result is an immediate consequence of (3.5) together with Lemma 3.1.

Lemma 3.2. Suppose that Assumption 1 holds. Let $\bar{z} \in L_{0}$ satisfy the MFCQ conditions (1.10) but not the KKT conditions (1.8). Then there exist positive quantities $R_{2}$ and $\epsilon$ such that for any $z \in \mathcal{B}\left(\bar{z}, R_{2}\right) \cap \mathcal{F}$ and any $\Delta>0$, we have

$$
\begin{aligned}
C(z, 1) & \geq \epsilon, \\
V(z, D, \Delta) & \geq \delta^{-1} \epsilon \min (1, \Delta),
\end{aligned}
$$

where $V(\cdot, \cdot, \cdot)$ is the negative of the value function for (3.4).

If Assumption 1 holds, we have that

$$
\left\|\Delta z^{\mathrm{L}}\right\|_{2} \leq \delta\left\|D \Delta z^{\mathrm{L}}\right\|_{p} \leq \delta \Delta .
$$

Hence, since $\Delta z$ is optimal for (3.3), and since $\Delta z^{\mathrm{L}}$ that solves (3.4) is feasible for this problem we have

$$
\begin{aligned}
& m(\Delta z) \leq m\left(\Delta z^{\mathrm{L}}\right) \\
& =\left(\Delta z^{\mathrm{L}}\right)^{T} \nabla f(z)+\frac{1}{2}\left(\Delta z^{\mathrm{L}}\right)^{T} H\left(\Delta z^{\mathrm{L}}\right) \\
& \leq-V(z, D, \Delta)+\frac{1}{2} \delta^{2}\|H\| \Delta^{2} \\
& \leq-\delta^{-1} \min (1, \Delta) C(z, 1)+\frac{1}{2} \delta^{2}\|H\| \Delta^{2}
\end{aligned}
$$

where the last inequality follows from (3.5).

We now define the Cauchy point for problem (3.3) as

$$
\Delta z^{\mathrm{C}}=\alpha^{\mathrm{C}} \Delta z^{\mathrm{L}},
$$

where

$$
\alpha^{\mathrm{C}}=\arg \min _{\alpha \in[0,1]} \alpha \nabla f(z)^{T} \Delta z^{\mathrm{L}}+\frac{1}{2} \alpha^{2}\left(\Delta z^{\mathrm{L}}\right)^{T} H \Delta z^{\mathrm{L}} .
$$

We show that $\Delta z^{\mathrm{C}}$ has the following property:

$$
m\left(\Delta z^{\mathrm{C}}\right) \leq-\frac{1}{2} C(z, 1) \min \left[\delta^{-1}, \delta^{-1} \Delta,\left(\delta^{4} \bar{\Delta}^{2}\|H\|_{2}\right)^{-1} C(z, 1)\right],
$$

where $\bar{\Delta}$ is defined in Algorithm FP-SQP. We prove (3.11) by considering two cases. First, when $\left(\Delta z^{\mathrm{L}}\right)^{T} H \Delta z^{\mathrm{L}} \leq 0$, we have $\alpha^{\mathrm{C}}=1$ in $(3.10)$ and hence $\Delta z^{\mathrm{C}}=\Delta z^{\mathrm{L}}$. Similarly to (3.8), but using $\left(\Delta z^{\mathrm{L}}\right)^{T} H \Delta z^{\mathrm{L}} \leq 0$ together with (3.5), we have

$$
m\left(\Delta z^{\mathrm{C}}\right)=m\left(\Delta z^{\mathrm{L}}\right) \leq-V(z, D, \Delta) \leq-\delta^{-1} C(z, 1) \min (1, \Delta),
$$


so the result (3.11) holds in this case. In the alternative case $\left(\Delta z^{\mathrm{L}}\right)^{T} H \Delta z^{\mathrm{L}}>0$, we have

$$
\alpha=\min \left(1, \frac{-\nabla f(z)^{T} \Delta z^{\mathrm{L}}}{\left(\Delta z^{\mathrm{L}}\right)^{T} H \Delta z^{\mathrm{L}}}\right)
$$

If the minimum is achieved at 1 , we have from $\left(\Delta z^{\mathrm{L}}\right)^{T} H \Delta z^{\mathrm{L}} \leq-\nabla f(z)^{T} \Delta z^{\mathrm{L}}$ and (3.5) that

$$
m\left(\Delta z^{\mathrm{C}}\right)=m\left(\Delta z^{\mathrm{L}}\right) \leq \frac{1}{2} \nabla f(z)^{T} \Delta z^{\mathrm{L}} \leq-\frac{1}{2} \delta^{-1} C(z, 1) \min (1, \Delta),
$$

and therefore again (3.11) is satisfied. If the min in (3.12) is achieved at $-\nabla f(z)^{T} \Delta z^{\mathrm{L}} /\left(\Delta z^{\mathrm{L}}\right)^{T} H \Delta z^{\mathrm{L}}$, we have from (3.5) that

$$
m\left(\Delta z^{\mathrm{C}}\right)=m\left(\alpha \Delta z^{\mathrm{L}}\right)=-\frac{1}{2} \frac{\left(\nabla f(z)^{T} \Delta z^{\mathrm{L}}\right)^{2}}{\left(\Delta z^{\mathrm{L}}\right)^{T} H \Delta z^{\mathrm{L}}} \leq-\frac{1}{2} \frac{\delta^{-2} \min \left(1, \Delta^{2}\right) C(z, 1)^{2}}{\|H\|_{2}\left\|\Delta z^{\mathrm{L}}\right\|_{2}^{2}} .
$$

Because of (3.7), we have from (3.14) that

$$
\begin{aligned}
& m\left(\Delta z^{\mathrm{C}}\right) \leq-\frac{1}{2} \frac{\delta^{-2} \min \left(1, \Delta^{2}\right) C(z, 1)^{2}}{\delta^{2} \Delta^{2}\|H\|_{2}} \\
& =-\frac{1}{2}\left(\delta^{4}\|H\|_{2}\right)^{-1} \min \left(1, \Delta^{-2}\right) C(z, 1)^{2} \leq-\frac{1}{2}\left(\delta^{4} \bar{\Delta}^{2}\|H\|_{2}\right)^{-1} C(z, 1)^{2},
\end{aligned}
$$

which again implies that (3.11) is satisfied.

Since $\Delta z^{\mathrm{C}}$ is feasible for (3.3), we have proved the following lemma.

Lemma 3.3. Suppose that $z \in L_{0}$ and that Assumption 1 holds. Suppose that $\Delta z^{C}$ is obtained from (3.4), (3.9), and (3.10). Then the decrease in the model function $m$ obtained by the point $\Delta z^{C}$ satisfies the bound (3.11), and therefore the solution $\Delta z$ of (3.3) satisfies the similar bound

$$
m(\Delta z) \leq-\frac{1}{2} C(z, 1) \min \left[\delta^{-1}, \delta^{-1} \Delta,\left(\delta^{4} \bar{\Delta}^{2}\|H\|_{2}\right)^{-1} C(z, 1)\right],
$$

where $C(z, 1)$ is the negative of the value function of $C L P(z, 1)$ defined in (3.1).

Note that this lemma holds even when we assume only that $\Delta z$ is feasible for (3.3) and satisfies $m(\Delta z) \leq m\left(\Delta z^{\mathrm{C}}\right)$. This relaxation is significant since, when $H$ is indefinite, the complexity of finding a solution of (3.3) is greater than the complexity of computing $\Delta z^{\mathrm{C}}$.

3.2. Result I: At Least One KKT Limit Point. We now discuss convergence of the sequence of iterates generated by the algorithm under the assumptions of Section 2, and the additional assumption that the Hessians $H_{k}$ of (1.2) are bounded as follows:

$$
\left\|H_{k}\right\|_{2} \leq \sigma_{0}+\sigma_{1} k, \quad k=0,1,2 \ldots
$$

The style of analysis follows that of a number of earlier works on convergence of trust-region algorithms for unconstrained, possibly nonsmooth problems; for example Yuan [17], Wright [16]. However, many modifications are needed to adapt the algorithms to constrained problems and to the algorithm of Section 2.

We first prove a key lemma as a preliminary to the global convergence result of this section. It finds a lower bound on the trust-region radii in the case that no subsequence of $\left\{C\left(z^{k}, 1\right)\right\}$ approaches zero. 
LEMMA 3.4. Suppose that Assumptions 1, 2, and 3 are satisfied, and that there are $\epsilon>0$ and an index $K$ such that

$$
C\left(z^{k}, 1\right) \geq \epsilon, \text { for all } k \geq K
$$

Then there is a constant $T>0$ such that

$$
\Delta_{k} \geq T / N_{k}, \text { for all } k \geq K
$$

where

$$
N_{k} \stackrel{\text { def }}{=} 1+\max _{i=0,1, \ldots, k}\left\|H_{k}\right\|_{2}
$$

Proof. For $\Delta_{k} \geq 1$, the claim (3.17) obviously holds with $T=1$. Hence, we assume for the remainder of the proof that $\Delta_{k} \in(0,1]$.

From Lemma 3.3, we have

$$
\begin{aligned}
-m_{k}\left(\Delta z^{k}\right) & \geq \frac{1}{2} \epsilon \min \left[\delta^{-1} \Delta_{k},\left(\delta^{4} \bar{\Delta}^{2}\left\|H_{k}\right\|_{2}\right)^{-1} \epsilon\right] \\
& \geq \frac{1}{2} \epsilon \min \left[\delta^{-1} \Delta_{k},\left(\delta^{4} \bar{\Delta}^{2} N_{k}\right)^{-1} \epsilon\right]
\end{aligned}
$$

We define the constants $\bar{\sigma}$ and $\gamma$ as follows:

$$
\bar{\sigma}=\sup \left\{\left\|\nabla^{2} f(z)\right\|_{2} \mid z \in \mathcal{N}\left(L_{0}\right)\right\}, \quad \gamma=\sup \left\{\|\nabla f(z)\|_{2} \mid z \in L_{0}\right\}
$$

where $\mathcal{N}\left(L_{0}\right)$ is the neighborhood defined in Assumption 2. Suppose now that $T$ is chosen small enough to satisfy the following conditions:

$$
\begin{aligned}
T & \leq 1 \\
\left\{z \mid \operatorname{dist}\left(z, L_{0}\right) \leq 2 \delta T\right\} & \subset \mathcal{N}\left(L_{0}\right) \\
2 T & \leq \epsilon /\left(\delta^{3} \bar{\Delta}^{2}\right), \\
(\gamma+2 \bar{\sigma} \delta) \phi(2 \delta T) \delta^{2} & \leq(1 / 48) \epsilon \\
2 \bar{\sigma} \delta^{3} T & \leq(1 / 48) \epsilon \\
\delta^{3} T & \leq(1 / 48) \epsilon
\end{aligned}
$$

where $\phi(\cdot)$ is defined in (1.5).

For any $k$ with

$$
\left\|\Delta z^{k}\right\| \leq 2 \delta T
$$

we have from Taylor's Theorem and the definition of $m_{k}$ that

$$
\begin{aligned}
& f\left(z^{k}\right)-f\left(z^{k}+\widetilde{\Delta z}^{k}\right)+m_{k}\left(\Delta z^{k}\right) \\
& =-\nabla f\left(z^{k}\right)^{T} \widetilde{\Delta z}^{k}-\frac{1}{2}(\widetilde{\Delta z})^{k} \nabla^{2} f\left(z_{\theta}^{k}\right) \widetilde{\Delta z}^{k}+\nabla f\left(z^{k}\right)^{T} \Delta z^{k}+\frac{1}{2}\left(\Delta z^{k}\right)^{T} H_{k} \Delta z^{k} \\
& =\left[\nabla f\left(z^{k}\right)+\nabla^{2} f\left(z_{\theta}^{k}\right) \Delta z^{k}\right]^{T}\left(\Delta z^{k}-\widetilde{\Delta z}^{k}\right) \\
& \quad-\frac{1}{2}\left(\widetilde{\Delta z}-\Delta z^{k}\right)^{T} \nabla^{2} f\left(z_{\theta}^{k}\right)\left(\widetilde{\Delta z}-\Delta z^{k}\right)-\frac{1}{2}\left(\Delta z^{k}\right)^{T}\left(\nabla^{2} f\left(z_{\theta}^{k}\right)-H_{k}\right) \Delta z^{k},
\end{aligned}
$$


where $z_{\theta}^{k}$ lies on the line segment between $z^{k}$ and $z^{k}+\widetilde{\Delta z}$. If $k$ is an index satisfying (3.21), we have from feasibility of both $z^{k}$ and $z^{k}+\widetilde{\Delta z}$ that

$$
\begin{aligned}
\operatorname{dist}\left(z_{\theta}^{k}, L_{0}\right) & \leq \frac{1}{2}\left\|\widetilde{\Delta z}^{k}\right\|_{2} \\
& \leq \frac{1}{2}\left(\left\|\Delta z^{k}\right\|_{2}+\left\|\Delta z^{k}-\widetilde{\Delta z}^{k}\right\|_{2}\right) \\
& \leq \frac{1}{2}\left(\left\|\Delta z^{k}\right\|_{2}+\phi\left(\left\|\Delta z^{k}\right\|_{2}\right)\left\|\Delta z^{k}\right\|_{2}\right) \\
& \leq \frac{1}{2}(2 \delta T+\phi(2 \delta T) 2 \delta T) \leq 2 \delta T,
\end{aligned}
$$

and therefore from (3.20b) and (3.19) we have $\left\|\nabla^{2} f\left(z_{\theta}^{k}\right)\right\|_{2} \leq \bar{\sigma}$. For $k$ satisfying (3.21), we have from (3.22) that

$$
\begin{aligned}
& \left|f\left(z^{k}\right)-f\left(z^{k}+\widetilde{\Delta z}^{k}\right)+m_{k}\left(\Delta z^{k}\right)\right| \\
& \leq\left(\left\|\nabla f\left(z^{k}\right)\right\|_{2}+\left\|\nabla^{2} f\left(z_{\theta}^{k}\right)\right\|_{2}\left\|\Delta z^{k}\right\|_{2}\right)\left\|\Delta z^{k}-\widetilde{\Delta z}^{k}\right\|_{2} \\
& \quad+\frac{1}{2}\left\|\nabla^{2} f\left(z_{\theta}^{k}\right)\right\|_{2}\left\|\widetilde{\Delta z}{ }^{k}-\Delta z^{k}\right\|_{2}^{2}+\frac{1}{2}\left(\left\|\nabla^{2} f\left(z_{\theta}^{k}\right)\right\|_{2}+\left\|H_{k}\right\|_{2}\right)\left\|\Delta z^{k}\right\|_{2}^{2} \\
& \leq(\gamma+2 \bar{\sigma} \delta T)\left\|\Delta z^{k}-\widetilde{\Delta z}^{k}\right\|_{2}+\frac{1}{2} \bar{\sigma}\left\|\Delta z^{k}-\widetilde{\Delta z}^{k}\right\|_{2}^{2}+\frac{1}{2}\left(\bar{\sigma}+N_{k}\right)\left\|\Delta z^{k}\right\|_{2}^{2} .
\end{aligned}
$$

Now using (1.5) and Assumption 1, we have for indices $k$ satisfying (3.21) that

$$
\begin{aligned}
& \left|f\left(z^{k}\right)-f\left(z^{k}+\widetilde{\Delta z}^{k}\right)+m_{k}\left(\Delta z^{k}\right)\right| \\
& \leq(\gamma+2 \bar{\sigma} \delta T) \phi\left(\left\|\Delta z^{k}\right\|_{2}\right)\left\|\Delta z^{k}\right\|_{2}+\frac{1}{2} \bar{\sigma} \phi\left(\left\|\Delta z^{k}\right\|_{2}\right)^{2}\left\|\Delta z^{k}\right\|_{2}^{2}+\frac{1}{2}\left(\bar{\sigma}+N_{k}\right)\left\|\Delta z^{k}\right\|_{2}^{2} \\
& \leq\left[(\gamma+2 \bar{\sigma} \delta T) \phi(2 \delta T)+\frac{1}{2} \bar{\sigma} \phi(2 \delta T)^{2} 2 \delta T+\bar{\sigma} \delta T+\frac{1}{2} N_{k}\left\|\Delta z^{k}\right\|_{2}\right]\left\|\Delta z^{k}\right\|_{2} \\
& \leq\left[(\gamma+2 \bar{\sigma} \delta T) \phi(2 \delta T)+\bar{\sigma} \delta T+\bar{\sigma} \delta T+\frac{1}{2} N_{k}\left\|\Delta z^{k}\right\|_{2}\right]\left\|\Delta z^{k}\right\|_{2} \\
& \leq\left[\frac{1}{48} \frac{\epsilon}{\delta^{2}}+\frac{1}{48} \frac{\epsilon}{\delta^{2}}+\frac{1}{2} N_{k}\left\|\Delta z^{k}\right\|_{2}\right]\left\|\Delta z^{k}\right\|_{2}
\end{aligned}
$$

where we used $\phi \leq 1 / 2,(3.20 \mathrm{~d})$, and (3.20e) to derive the various inequalities.

Now suppose that (3.17) is not satisfied for all $k$ and for our choice of $T$, and suppose that $l$ is the first index at which it is violated, that is,

$$
\Delta_{l}<T / N_{l} .
$$

We exclude the case $l=K$ (by decreasing $T$ further, if necessary), and consider the index $l-1$. Since $\Delta_{k} \geq(1 / 2)\left\|D_{k-1} \Delta z^{k-1}\right\|_{p}$ for all $k$, and since $N_{l} \geq 1$, we have

$$
\left\|\Delta z^{l-1}\right\|_{2} \leq \delta\left\|D_{l-1} \Delta z^{l-1}\right\|_{p} \leq 2 \delta \Delta_{l}<2 \delta T,
$$

so that $l-1$ satisfies (3.21). Hence, the bound (3.24) applies with $k=l-1$, and we have

$$
\begin{aligned}
& \left|f\left(z^{l-1}\right)-f\left(z^{l-1}+\widetilde{\Delta z}{ }^{l-1}\right)+m_{l-1}\left(\Delta z^{l-1}\right)\right| \\
& \leq\left[\frac{1}{24} \frac{\epsilon}{\delta^{2}}+\frac{1}{2} N_{l-1}\left\|\Delta z^{l-1}\right\|_{2}\right]\left\|\Delta z^{l-1}\right\|_{2} .
\end{aligned}
$$

Since $N_{l-1} \leq N_{l}$, we have from (3.26) and (3.25) that

$$
N_{l-1}\left\|\Delta z^{l-1}\right\|_{2} \leq 2 \delta N_{l} \Delta_{l}<2 \delta T \text {. }
$$


Therefore by using (3.27) and (3.20f), we obtain

$$
\begin{aligned}
& \left|f\left(z^{l-1}\right)-f\left(z^{l-1}+\widetilde{\Delta z}{ }^{l-1}\right)+m_{l-1}\left(\Delta z^{l-1}\right)\right| \\
& \leq\left(\frac{1}{24} \frac{\epsilon}{\delta^{2}}+\delta T\right)\left\|\Delta z^{l-1}\right\|_{2} \leq \frac{1}{16} \frac{\epsilon}{\delta^{2}}\left\|\Delta z^{l-1}\right\|_{2} .
\end{aligned}
$$

Returning to the right-hand side of (3.18), we have for $k=l-1$ that

$$
\delta^{-1} \Delta_{l-1} \geq \delta^{-1}\left\|D_{l-1} \Delta z^{l-1}\right\|_{p} \geq \delta^{-2}\left\|\Delta z^{l-1}\right\|_{2},
$$

and using (3.28) and (3.20c), we have

$$
\frac{\epsilon}{\delta^{4} \bar{\Delta}^{2} N_{l-1}} \geq \frac{\epsilon}{\delta^{4} \bar{\Delta}^{2}} \frac{\left\|\Delta z^{l-1}\right\|_{2}}{2 \delta T} \geq \delta^{-2}\left\|\Delta z^{l-1}\right\|_{2} .
$$

Hence, from (3.18) and the last two inequalities, we have

$$
-m_{l-1}\left(\Delta z^{l-1}\right) \geq \frac{1}{2} \frac{\epsilon}{\delta^{2}}\left\|\Delta z^{l-1}\right\|_{2} .
$$

By comparing (3.29) and (3.30), we have from (2.1) that

$$
\begin{aligned}
\rho_{l-1} & =\frac{f\left(z^{l-1}\right)-f\left(z^{l-1}+\widetilde{\Delta z}^{l-1}\right)}{-m_{l-1}\left(\Delta z^{l-1}\right)} \\
& \geq 1-\frac{\left|f\left(z^{l-1}\right)-f\left(z^{l-1}+\widetilde{\Delta z}^{l-1}\right)+m_{l-1}\left(\Delta z^{l-1}\right)\right|}{-m_{l-1}\left(\Delta z^{l-1}\right)} \\
& \geq 1-\frac{1}{8}=\frac{7}{8} .
\end{aligned}
$$

Hence, by the workings of the algorithm, we have $\Delta_{l} \geq \Delta_{l-1}$. But since $N_{l-1} \leq N_{l}$, we have $N_{l-1} \Delta_{l-1} \leq N_{l} \Delta_{l}$, so that $\Delta_{l-1}<T / N_{l-1}$, which contradicts the definition of $l$ as the first index that violates (3.17). We conclude that no such $l$ exists, and hence that (3.17) holds. $\square$

The following technical lemma, attributed to M. J. D. Powell, is proved in Yuan $[17$, Lemma 3.4]. We modify the statement slightly to start the sequence at the index $K$ rather than at 0 .

Lemma 3.5. Suppose $\left\{\Delta_{k}\right\}$ and $\left\{N_{k}\right\}$ are two sequences such that $\Delta_{k} \geq T / N_{k}$ for all $k \geq K$, for some integer $K$ and constant $T>0$. Let $\mathcal{K} \subset\{K, K+1, K+2, \ldots\}$ be defined such that

$$
\begin{aligned}
\Delta_{k+1} & \leq \tau_{0} \Delta_{k} \text { if } k \in \mathcal{K}, \\
\Delta_{k+1} & \leq \tau_{1} \Delta_{k} \text { if } k \notin \mathcal{K}, \\
N_{k+1} & \geq N_{k} \text { for all } k \geq K, \\
\sum_{k \in \mathcal{K}} \min \left(\Delta_{k}, 1 / N_{k}\right) & <\infty,
\end{aligned}
$$

where $\tau_{0}$ and $\tau_{1}$ are constants satisfying $0<\tau_{1}<1<\tau_{0}$. Then

$$
\sum_{k=K}^{\infty} 1 / N_{k}<\infty .
$$


Our main global convergence result for this section is as follows.

THEOREM 3.6. Suppose that Assumptions 1, 2, and 3 are satisfied, and that the approximate Hessians $H_{k}$ satisfy (3.16); that is, $\left\|H_{k}\right\|_{2} \leq \sigma_{0}+k \sigma_{1}$, for some nonnegative constants $\sigma_{0}$ and $\sigma_{1}$. Then Algorithm FP-SQP either terminates at a KKT point, or else it has at least one limit point which is either a KKT point or else fails to satisfy the MFCQ conditions (1.10).

Proof. Consider first the case in which the algorithm terminates finitely at some iterate $z^{k}$ at which $m_{k}\left(\Delta z^{k}\right)=0$. Then $\Delta z=0$ is a solution of the subproblem (1.2), (1.3) at $z=z^{k}$ at which the trust-region bound is inactive. The KKT conditions for the subproblem at $\Delta z=0$ correspond exactly to the KKT conditions (1.8) for the original problem (1.1) at $z^{k}$.

In the alternative case, the algorithm generates an infinite sequence $\left\{z^{k}\right\}$. Suppose first that it is possible to choose $\epsilon>0$ and $K$ such that the conditions of Lemma 3.4 are satisfied. We apply Lemma 3.5 , choosing $\mathcal{K}$ to be the subsequence of $\{K, K+$ $1, K+2, \ldots\}$ at which the trust-region radius is not reduced. We can then set $\tau_{0}=2$, $\tau_{1}=0.5$, and define $N_{k}$ as in Lemma 3.4. At the iterates $k \in \mathcal{K}$, the algorithm takes a step, and we have $\rho_{k} \geq \eta$. By using (3.15) and (3.18), we then have

$$
\begin{aligned}
f\left(z^{k}\right)-f\left(z^{k}+\widetilde{\Delta z}^{k}\right) & \geq-\eta m_{k}\left(\Delta z^{k}\right) \\
& \geq \frac{1}{2} \eta \epsilon \min \left(\delta^{-1}, \delta^{-1} \Delta_{k}, \delta^{-4} \bar{\Delta}^{-2} \epsilon / N_{k}\right) \\
& \geq \frac{1}{2} \eta \epsilon \min \left(\delta^{-1}, \delta^{-4} \bar{\Delta}^{-2} \epsilon\right) \min \left(\Delta_{k}, 1 / N_{k}\right),
\end{aligned}
$$

where the final inequality follows from $N_{k} \geq 1$. By summing both sides of this inequality over $k \in \mathcal{K}$, and using the fact that $f\left(z^{k}\right)$ is bounded below (since $f$ is continuous on the compact level set $\left.L_{0}\right)$ we have that condition (3.31d) is satisfied. The conclusion (3.32) then holds. However, since from (3.16) we have $N_{k} \leq 1+\sigma_{0}+$ $\sigma_{1} k,(3.32)$ cannot hold, so we have a contradiction. We conclude therefore that it is not possible to choose $\epsilon>0$ and $K$ satisfying the conditions of Lemma 3.4, that is, there is a subsequence $\mathcal{J} \subset\{0,1,2, \ldots\}$ such that

$$
\lim _{k \in \mathcal{J}} C\left(z^{k}, 1\right)=0 .
$$

Since the points $z^{k}, k \in \mathcal{J}$ all belong to the compact set $L_{0}$, we can identify a limit point $\bar{z}$, and assume without loss of generality that $\lim _{k \in \mathcal{J}} z^{k}=\bar{z}$. From the observation immediately following the statement of Lemma 3.1, we have that either MFCQ conditions (1.10) fail to hold at $\bar{z}$, or else that $\bar{z}$ satisfies both the MFCQ conditions and the KKT conditions (1.8).

3.3. Result II: All Limit Points are KKT Points. In this section, we replace the bound (3.16) on the Hessians $H_{k}$ by a uniform bound

$$
\left\|H_{k}\right\|_{2} \leq \sigma
$$

for some constant $\sigma$, and obtain a stronger global convergence result; namely, that every limit point of the algorithm either fails to satisfy MFCQ or else is a KKT point.

As a preliminary to the main result of this section, we show that for any limit point $\bar{z}$ of Algorithm FP-SQP at which MFCQ but not KKT conditions are satisfied, there is a subsequence $\mathcal{K}$ with $z^{k} \rightarrow_{k \in \mathcal{K}} \bar{z}$ and $\Delta_{k} \rightarrow_{k \in \mathcal{K}} 0$.

Lemma 3.7. Suppose that Assumptions 1, 2, and 3 are satisfied, and that the Hessians $H_{k}$ satisfy the bound (3.33) for some $\sigma>0$. Suppose that $\bar{z}$ is a limit point of 
the sequence $\left\{z^{k}\right\}$ such that the MFCQ condition (1.10) holds but the KKT conditions (1.8) are not satisfied at $\bar{z}$. Then there exists an (infinite) subsequence $\mathcal{K}$ such that

$$
\lim _{k \in \mathcal{K}} z^{k}=\bar{z}
$$

and

$$
\lim _{k \in \mathcal{K}} \Delta_{k}=0
$$

Proof. Since $\bar{z} \in L_{0}$, we can define $\epsilon$ and $R_{2}$ as in Lemma 3.1. From this lemma, we have that $C(z, 1) \geq \epsilon$ for all $z \in \mathcal{B}\left(\bar{z}, R_{2}\right) \cap \mathcal{F}$. Hence, for such $z$, we have from Lemma 3.3 that the solution $\Delta z$ of the trust-region subproblem at (3.3) with $\Delta \in(0,1]$ satisfies

$$
\begin{aligned}
m(\Delta z) & \leq-\frac{1}{2} C(z, 1) \min \left[\delta^{-1}, \delta^{-1} \Delta,\left(\delta^{4} \bar{\Delta}^{2}\|H\|_{2}\right)^{-1} C(z, 1)\right] \\
& \leq-\frac{1}{2} \epsilon \min \left[\delta^{-1}, \delta^{-1} \Delta,\left(\delta^{4} \bar{\Delta}^{2} \sigma\right)^{-1} \epsilon\right],
\end{aligned}
$$

where we used the bound (3.33) to obtain the second inequality.

Because $\bar{z}$ is a limit point, we can certainly choose a subsequence $\mathcal{K}$ satisfying (3.34). By deleting the elements from $\mathcal{K}$ for which $z_{k} \notin \mathcal{B}\left(\bar{z}, R_{2}\right)$, we have from (3.36) that

$$
m_{k}\left(\Delta z^{k}\right) \leq-\frac{1}{2} \epsilon \min \left[\delta^{-1}, \delta^{-1} \Delta_{k},\left(\delta^{4} \bar{\Delta}^{2} \sigma\right)^{-1} \epsilon\right], \text { for all } k \in \mathcal{K} .
$$

We prove the result (3.35) by modifying $\mathcal{K}$ and taking further subsequences as necessary. Consider first the case in which $\left\{z^{k}\right\}_{k \in \mathcal{K}}$ takes on only a finite number of distinct values. We then must have that $z^{k}=\bar{z}$ for all $k \in \mathcal{K}$ sufficiently large. Now, remove from $\mathcal{K}$ all indices $k$ for which $z^{k} \neq \bar{z}$. Suppose for contradiction that some subsequent iterate in the full sequence $\left\{z^{k}\right\}$ is different from $\bar{z}$. If $\bar{k} \geq k$ is some iterate such that

$$
f\left(z^{\bar{k}}\right)<f\left(z^{k}\right)=f(\bar{z}),
$$

we have by monotonicity of $\left\{f\left(z^{l}\right)\right\}$ (for the full sequence of function values) that

$$
f\left(z^{l}\right) \leq f\left(z^{\bar{k}}\right)<f(\bar{z})
$$

for all $l>\bar{k}$. Hence the function values in the tail of the full sequence are bounded away from $f(\bar{z})$, so it is not possible to choose a subsequence $\mathcal{K}$ with the property (3.34). Therefore, we have that $z^{l}=\bar{z}$ for all $l \geq k$, so that all steps generated by Algorithm FP-SQP after iteration $k$ fail the acceptance condition. We then have that

$$
\Delta_{l+1}=\frac{1}{2}\left\|D_{l} \Delta z^{l}\right\|_{p} \leq \frac{1}{2} \Delta_{l}, \text { for all } l \geq k,
$$

so that $\Delta_{l} \rightarrow 0$ as $l \rightarrow \infty$ (for the full sequence). Hence, in particular, (3.35) holds.

We consider now the second case, in which $\left\{z^{k}\right\}_{k \in \mathcal{K}}$ takes on an infinite number of distinct values. Without loss of generality, we can assume that all elements $z^{k}$, $k \in \mathcal{K}$ are distinct (by dropping the repeated elements if necessary). Moreover, we can assume that $z^{k+1} \neq z^{k}$ for all $k \in \mathcal{K}$, by replacing $k$ if necessary by the largest index $\bar{k}$ such that $\bar{k} \geq k$ and $z^{\bar{k}}=z^{k}$. Thus, we have that the sufficient decrease 
condition $\rho_{k} \geq \eta$ is satisfied at all $k \in \mathcal{K}$. Therefore from (2.1) and (3.36), and the easily demonstrated fact that $f\left(z^{l}\right) \geq f(\bar{z})$ for all $l=0,1,2, \ldots$, we have

$$
\begin{aligned}
f\left(z^{k}\right)-f(\bar{z}) & \geq f\left(z^{k}\right)-f\left(z^{k+1}\right) \\
& \geq-\eta m_{k}\left(\Delta z^{k}\right) \\
& \geq \frac{1}{2} \eta \epsilon \min \left[\delta^{-1}, \delta^{-1} \Delta_{k},\left(\delta^{4} \bar{\Delta}^{2} \sigma\right)^{-1} \epsilon\right] \geq 0 .
\end{aligned}
$$

Since $f\left(z^{k}\right) \rightarrow_{k \in \mathcal{K}} f(\bar{z})$, we have from this chain of inequalities that (3.35) is satisfied in this case too. Hence, we have demonstrated (3.35).

We now prove the main global convergence result of this section.

TheOREM 3.8. Suppose that Assumptions 1, 2, and 3 are satisfied, and that the Hessian approximations $H_{k}$ satisfy (3.33). Then all limit points of Algorithm FP-SQP either are KKT points or else fail to satisfy the MFCQ conditions (1.10).

Proof. Suppose for contradiction that $\bar{z}$ is a limit point at which (1.10) holds but (1.8) are not satisfied, and let $R_{2}$ and $\epsilon$ be defined as in the proof of Lemma 3.7. We invoke Lemma 3.7 to define the subsequence $\mathcal{K}$ with the properties (3.34) and (3.35). The inequality (3.37) also holds for the subsequence $\mathcal{K}$.

Let $\bar{\sigma}$ and $\gamma$ be defined as in (3.19). We now define the constants $R>0$ and $\Delta_{\phi}>0$ such that that the following conditions hold:

$$
\begin{aligned}
R & \leq R_{2}, \\
\gamma \phi\left(\Delta_{\phi}\right) & \leq \frac{1}{16} \frac{\epsilon}{\delta^{2}}, \\
\mathcal{B}\left(\bar{z}, R+\Delta_{\phi}\right) \cap \mathcal{F} & \subset \mathcal{N}\left(L_{0}\right), \\
\Delta_{\phi} & \leq \Delta_{\text {def }},
\end{aligned}
$$

where $\Delta_{\text {def }}$ is defined in Theorem 2.2. Note in particular from the latter theorem that $\widetilde{\Delta z}$ satisfying (1.4) and (1.5) exists whenever $\|D \Delta z\|_{2} \leq \Delta_{\phi}$.

Given $R$ and $\Delta_{\phi}$, we can now define $\tilde{\Delta}>0$ small enough to satisfy the following properties:

$$
\begin{aligned}
\tilde{\Delta} & \leq 1, \\
\left(2 \bar{\sigma}+\frac{1}{2} \sigma\right) \delta \tilde{\Delta} & \leq \frac{1}{16} \frac{\epsilon}{\delta^{2}}, \\
\tilde{\Delta} & \leq \frac{2 \Delta_{\phi}}{3 \delta}, \\
\tilde{\Delta} & \leq \frac{\epsilon}{\delta^{3} \bar{\Delta}^{2} \sigma},
\end{aligned}
$$

where $\bar{\Delta}$ is the overall upper bound on trust-region radius. We then define $\hat{\epsilon}>0$ as follows:

$$
\hat{\epsilon}=\frac{1}{2} \eta \epsilon \min \left(\delta^{-1}, \frac{1}{4} R / \delta^{2},\left(\delta^{4} \bar{\Delta}^{2} \sigma\right)^{-1} \epsilon\right) .
$$

Finally, we define an index $q \in \mathcal{K}$ sufficient large that

$$
\begin{aligned}
\left\|z^{q}-\bar{z}\right\|_{2} & <R / 2, \\
f\left(z^{q}\right)-f(\bar{z}) & \leq \hat{\epsilon} / 2 .
\end{aligned}
$$

(Existence of such an index $q$ follows immediately from $z^{k} \rightarrow \mathcal{K} \bar{z}$.) 
Consider the neighborhood

$$
\operatorname{cl}\left(\mathcal{B}\left(z^{q}, R / 2\right)\right) \cap \mathcal{F},
$$

which is contained in $\mathcal{B}(\bar{z}, R) \cap \mathcal{F}$ because of (3.41a). We consider two cases.

Case I: All remaining iterates $z^{q+1}, z^{q+2}, \ldots$ of the full sequence remain inside the neighborhood (3.42). If

$$
\left\|D_{k} \Delta z^{k}\right\|_{p} \leq \tilde{\Delta}, \text { for any } k=q, q+1, q+2, \ldots,
$$

we have from $(1.6)$ and $(3.39 \mathrm{c})$ that

$$
\|\widetilde{\Delta z}\|_{2} \leq(3 / 2)\left\|\Delta z^{k}\right\|_{2} \leq(3 / 2) \delta\left\|D_{k} \Delta z^{k}\right\|_{p} \leq(3 / 2) \delta \tilde{\Delta} \leq \Delta_{\phi} .
$$

We now show that whenever (3.43) occurs, the ratio $\rho_{k}$ defined by $(2.1)$ is at least $3 / 4$, so that the trust-region radius $\Delta_{k+1}$ for the next iteration is no smaller than the one for this iteration, $\Delta_{k}$. As in the proof of Lemma 3.4, the relation (3.22) holds, with $z_{\theta}^{k}$ satisfying

$$
\operatorname{dist}\left(z_{\theta}^{k}, L_{0}\right) \leq \frac{1}{2}\|\widetilde{\Delta z}\|_{2}^{k} \leq \frac{1}{2} \Delta_{\phi} .
$$

Hence, from (3.19) and (3.38c), we have $\left\|\nabla^{2} f\left(z_{\theta}^{k}\right)\right\|_{2} \leq \bar{\sigma}$. Similarly to (3.23), we have

$$
\begin{aligned}
& \left|f\left(z^{k}\right)-f\left(z^{k}+\widetilde{\Delta z}{ }^{k}\right)+m_{k}\left(\Delta z^{k}\right)\right| \\
& \leq\left(\left\|\nabla f\left(z^{k}\right)\right\|_{2}+\left\|\nabla^{2} f\left(z_{\theta}^{k}\right)\right\|_{2}\left\|\Delta z^{k}\right\|_{2}\right)\left\|\Delta z^{k}-\widetilde{\Delta z}^{k}\right\|_{2} \\
& \quad \quad+\frac{1}{2}\left\|\nabla^{2} f\left(z_{\theta}^{k}\right)\right\|_{2}\left\|\widetilde{\Delta z}-\Delta z^{k}\right\|_{2}^{2}+\frac{1}{2}\left(\left\|\nabla^{2} f\left(z_{\theta}^{k}\right)\right\|_{2}+\left\|H_{k}\right\|_{2}\right)\left\|\Delta z^{k}\right\|_{2}^{2} \\
& \leq(\gamma+\bar{\sigma} \delta \tilde{\Delta}) \phi\left(\left\|\Delta z^{k}\right\|_{2}\right)\left\|\Delta z^{k}\right\|_{2}+\frac{1}{2} \bar{\sigma} \phi\left(\left\|\Delta z^{k}\right\|_{2}\right)^{2}\left\|\Delta z^{k}\right\|_{2}^{2}+\frac{1}{2}(\bar{\sigma}+\sigma)\left\|\Delta z^{k}\right\|_{2}^{2},
\end{aligned}
$$

where we used (3.19) and $\left\|\Delta z^{k}\right\|_{2} \leq \delta \tilde{\Delta}$ from (3.44) in deriving the second inequality. Now using (3.44) again, together with monotonicity of $\phi, \phi(\cdot) \leq 1 / 2,(3.38 \mathrm{~b})$, and (3.39b), we have

$$
\begin{aligned}
& \left|f\left(z^{k}\right)-f\left(z^{k}+\widetilde{\Delta z^{k}}\right)+m_{k}\left(\Delta z^{k}\right)\right| \\
& \leq(\gamma+\bar{\sigma} \delta \tilde{\Delta}) \phi\left(\Delta_{\phi}\right)\left\|\Delta z^{k}\right\|_{2}+\left[\frac{1}{2} \bar{\sigma} \phi\left(\Delta_{\phi}\right)^{2} \delta \tilde{\Delta}+\frac{1}{2}(\bar{\sigma}+\sigma) \delta \tilde{\Delta}\right]\left\|\Delta z^{k}\right\|_{2} \\
& \leq\left[\gamma \phi\left(\Delta_{\phi}\right)+\left(\bar{\sigma} \delta \tilde{\Delta}+\frac{1}{2} \bar{\sigma} \delta \tilde{\Delta}+\frac{1}{2}(\bar{\sigma}+\sigma) \delta \tilde{\Delta}\right)\right]\left\|\Delta z^{k}\right\|_{2} \\
& =\left[\gamma \phi\left(\Delta_{\phi}\right)+\left(2 \bar{\sigma}+\frac{1}{2} \sigma\right) \delta \tilde{\Delta}\right]\left\|\Delta z^{k}\right\|_{2} \\
& \leq\left(\frac{1}{16} \frac{\epsilon}{\delta^{2}}+\frac{1}{16} \frac{\epsilon}{\delta^{2}}\right)\left\|\Delta z^{k}\right\|_{2}=\frac{1}{8} \frac{\epsilon}{\delta^{2}}\left\|\Delta z^{k}\right\|_{2} .
\end{aligned}
$$

Meanwhile, from (3.36) and since $z^{k} \in \mathcal{B}(\bar{z}, R) \cap \mathcal{F}$ where $R \leq R_{2}$, we have

$$
-m_{k}\left(\Delta z^{k}\right) \geq \frac{1}{2} \epsilon \min \left(\delta^{-1}, \delta^{-1} \Delta_{k},\left(\delta^{4} \bar{\Delta}^{2} \sigma\right)^{-1} \epsilon\right) .
$$

Now from Assumption 1 we have

$$
\Delta_{k} \geq\left\|D_{k} \Delta z^{k}\right\|_{p} \geq \delta^{-1}\left\|\Delta z^{k}\right\|_{2},
$$


while from (3.39a) and (3.43), we have

$$
1 \geq \tilde{\Delta} \geq\left\|D_{k} \Delta z^{k}\right\|_{p} \geq \delta^{-1}\left\|\Delta z^{k}\right\|_{2} .
$$

From (3.39d) and Assumption 1, we have

$$
\epsilon \geq \delta^{3} \bar{\Delta}^{2} \sigma \tilde{\Delta} \geq \delta^{3} \bar{\Delta}^{2} \sigma\left\|D_{k} \Delta z^{k}\right\|_{p} \geq \delta^{2} \bar{\Delta}^{2} \sigma\left\|\Delta z^{k}\right\|_{2} .
$$

By substituting these last three expressions into (3.46), we obtain

$$
-m_{k}\left(\Delta z^{k}\right) \geq \frac{1}{2} \frac{\epsilon}{\delta^{2}}\left\|\Delta z^{k}\right\|_{2} .
$$

We then have from (2.1), and using (3.45) and (3.47), that

$$
\begin{aligned}
\rho_{k} & =\frac{f\left(z^{k}\right)-f\left(z^{k}+\widetilde{\Delta z}^{k}\right)}{-m_{k}\left(\Delta z^{k}\right)} \\
& \geq 1-\frac{\left|f\left(z^{k}\right)-f\left(z^{k}+\widetilde{\Delta z}{ }^{k}\right)+m_{k}\left(\Delta z^{k}\right)\right|}{-m_{k}\left(\Delta z^{k}\right)} \\
& \geq 3 / 4 .
\end{aligned}
$$

It follows that the algorithm sets

$$
\Delta_{k+1} \geq \Delta_{k}
$$

for all $k$ satisfying (3.43). For $k=q, q+1, q+2, \ldots$ not satisfying (3.43), Algorithm FP-SQP indicates that we may have reduction of the trust-region radius to

$$
\Delta_{k+1}=(1 / 2)\left\|D_{k} \Delta z^{k}\right\|_{p} \geq(1 / 2) \tilde{\Delta} .
$$

By considering both cases, we conclude that

$$
\Delta_{k} \geq \min \left(\Delta_{q},(1 / 2) \tilde{\Delta}\right), \text { for all } k=q, q+1, q+2, \ldots,
$$

which contradicts (3.35). Hence, Case I cannot occur.

We now consider the alternative.

Case II: Some subsequent iterate $z^{q+1}, z^{q+2}, \ldots$ leaves the neighborhood (3.42). If $z^{l}$ is the first iterate outside this neighborhood, note that all iterates $z^{k}, k=q, q+1, q+$ $2, \ldots, l-1$ lie inside the set $\mathcal{B}(\bar{z}, R) \cap \mathcal{F}$, within which (3.36) applies. By summing over the "successful" iterates in this span, we have the following:

$$
\begin{aligned}
& f\left(z^{q}\right)-f\left(z^{l}\right) \\
& =\sum_{\substack{k=q \\
z^{k \neq z^{k+1}}}}^{l-1} f\left(z^{k}\right)-f\left(z^{k+1}\right) \\
& \geq \sum_{\substack{k=q \\
z^{k \neq z^{k+1}}}}^{l-1}-\eta m_{k}\left(\Delta z^{k}\right) \quad \text { by }(2.1) \text { and Algorithm FP-SQP } \\
& \geq \eta \sum_{\substack{k=q \\
z^{k} \neq z^{k+1}}}^{l-1} \frac{1}{2} \epsilon \min \left[\delta^{-1}, \delta^{-1} \Delta_{k},\left(\delta^{4} \bar{\Delta}^{2} \sigma\right)^{-1} \epsilon\right] \quad \text { by }(3.36) \\
& \geq \frac{1}{2} \eta \epsilon \min \\
& \left.\delta^{-1}, \delta^{-1} \sum_{\substack{k=q \\
z^{k} \neq z^{k+1}}}^{l-1} \Delta_{k},\left(\delta^{4} \bar{\Delta}^{2} \sigma\right)^{-1} \epsilon\right] .
\end{aligned}
$$


We have from Assumption 1 and (1.6) that

$$
\Delta_{k} \geq\left\|D_{k} \Delta z^{k}\right\|_{p} \geq \delta^{-1}\left\|\Delta z^{k}\right\|_{2} \geq \frac{1}{2} \delta^{-1}\|\widetilde{\Delta z}\|_{2}
$$

so that (3.50) becomes

$$
f\left(z^{q}\right)-f\left(z^{l}\right) \geq \frac{1}{2} \eta \epsilon \min \left[\delta^{-1}, \sum_{\substack{k=q \\ z^{k} \neq z^{k+1}}}^{l-1} \frac{1}{2} \delta^{-2}\|\widetilde{\Delta z}\|_{2},\left(\delta^{4} \bar{\Delta}^{2} \sigma\right)^{-1} \epsilon\right] .
$$

However, because $z^{l}$ lies outside the neighborhood (3.42) we have that

$$
R / 2 \leq\left\|z^{q}-z^{l}\right\|_{2} \leq \sum_{\substack{k=q \\ z^{k} \neq z^{k+1}}}^{l-1}\|\widetilde{\Delta z}\|_{2}^{k}
$$

so that (3.51) becomes

$$
f\left(z^{q}\right)-f\left(z^{l}\right) \geq \frac{1}{2} \eta \epsilon \min \left[\delta^{-1}, \frac{1}{4} \delta^{-2} R,\left(\delta^{4} \bar{\Delta}^{2} \sigma\right)^{-1} \epsilon\right] .
$$

By using this estimate together with the definition of $\hat{\epsilon}(3.40)$, we have

$$
f\left(z^{q}\right)-f\left(z^{l}\right) \geq \hat{\epsilon}
$$

But since $f\left(z^{l}\right) \geq f(\bar{z})$ (since $\bar{z}$ is a limit point of the full sequence), this inequality contradicts (3.41b). Hence, Case II cannot occur either, and the proof is complete.

4. Local Convergence. We now examine local convergence behavior of the algorithm to a point $z^{*}$ satisfying second-order sufficient conditions for optimality, under the assumption that $z^{k} \rightarrow z^{*}$. We do not attempt to obtain the most general possible superlinear convergence result, but rather make the kind of assumptions that are typically made in the local convergence analysis of SQP methods in which second derivatives of the objective and constraint functions are available. We also make additional assumptions on the feasibility perturbation process that is used to recover $\widetilde{\Delta z}^{k}$ from $\Delta z^{k}$. Ultimately, we show that Algorithm FP-SQP converges Qsuperlinearly.

We assume a priori that $z^{*}$ satisfies the KKT conditions, and define the active set $\mathcal{A}^{*}$ as follows:

$$
\mathcal{A}^{*} \stackrel{\text { def }}{=} \mathcal{A}\left(z^{*}\right)
$$

where $\mathcal{A}(\cdot)$ is defined in (1.9). In this section, we use the following subvector notation:

$$
d_{\mathcal{I}}(z) \stackrel{\text { def }}{=}\left[d_{i}(z)\right]_{i \in \mathcal{I}}, \quad \text { where } \mathcal{I} \subset\{1,2, \ldots, r\} .
$$

Assumption 4.

(a) The functions $f$, $c$, and $d$ are twice continuously differentiable in a neighborhood of $z^{*}$.

(b) The linear independence constraint qualification (LICQ) (1.11) is satisfied at $z^{*}$. 
(c) Strict complementarity holds; that is, for the (unique) multipliers $\left(\mu^{*}, \lambda^{*}\right)$ satisfying the KKT conditions (1.8) at $z=z^{*}$, we have $\lambda_{i}^{*}>0$ for all $i \in \mathcal{A}^{*}$.

(d) Second-order sufficient conditions are satisfied at $z^{*}$; that is, there is $\alpha>0$ such that

$$
v^{T} \nabla_{z z}^{2} \mathcal{L}\left(z^{*}, \mu^{*}, \lambda^{*}\right) v \geq \alpha\|v\|^{2}, \quad \forall v \text { s.t. } \nabla c\left(z^{*}\right)^{T} v=0, \quad \nabla d_{\mathcal{A}^{*}}\left(z^{*}\right)^{T} v=0,
$$

where the Lagrangian function $\mathcal{L}$ is defined in (1.7).

Besides these additional assumptions on the nature of the limit point $z^{*}$, we make additional assumptions on the algorithm itself. As mentioned above, we start by assuming that $z^{k} \rightarrow z^{*}$. We further assume that estimates $\mathcal{W}_{k}$ of the active set $\mathcal{A}^{*}$ and estimates $\left(\mu^{k}, \lambda^{k}\right)$ of the optimal Lagrange multipliers $\left(\mu^{*}, \lambda^{*}\right)$ are calculated at each iteration $k$, and that these estimates are asymptotically exact. It is known (see, for example, Facchinei, Fischer, and Kanzow [6]) that an asymptotically exact estimate $\mathcal{W}_{k}$ of $\mathcal{A}^{*}$ is available, given that $\left(z^{k}, \mu^{k}, \lambda^{k}\right) \rightarrow\left(z^{*}, \mu^{*}, \lambda^{*}\right)$, under weaker conditions than assumed here. On the other hand, it is also known that given an asymptotically exact $\mathcal{W}_{k}$, we can use a least-squares procedure to compute an asymptotically exact estimate $\left(\mu^{k}, \lambda^{k}\right)$ of $\left(\mu^{*}, \lambda^{*}\right)$. However, the simultaneous estimation of $\mathcal{W}_{k}$ and $\left(\mu^{k}, \lambda^{k}\right)$ is less straightforward. We anticipate, however, that a procedure that works well in practice would be relatively easy to implement, especially under the LICQ and strict complementarity assumptions. Given an initial guess of $\mathcal{W}_{k}$, such a procedure would alternate between a least-squares estimate of $\left(\mu^{k}, \lambda^{k}\right)$ and an active-set identification procedure like those in [6], until the estimate of $\mathcal{W}_{k}$ settles down. We note that the multipliers for the linearized constraints in the subproblem (1.2), (1.3) (denoted in the analysis below by $\bar{\mu}^{k}$ and $\bar{\lambda}^{k}$ ) do not necessarily satisfy the asymptotic exactness condition, unless it is known a priori that the trust region is inactive for all $k$ sufficiently large. Fletcher and Sainz de la Maza [7] have analyzed the behavior of these multipliers in the context of a sequential linear programming algorithm and show that, under certain assumptions, $\left(\mu^{*}, \lambda^{*}\right)$ is a limit point of the sequence $\left\{\left(\bar{\mu}^{k}, \bar{\lambda}^{k}\right)\right\}$.

We summarize the algorithmic assumptions as follows.

Assumption 5.

(a) $z^{k} \rightarrow z^{*}$.

(b) $\mathcal{W}_{k}=\mathcal{A}^{*}$ for all $k$ sufficiently large, where $\mathcal{W}_{k}$ is the estimate of the optimal active set.

(c) $\left(\mu^{k}, \lambda^{k}\right) \rightarrow\left(\mu^{*}, \lambda^{*}\right)$.

(d) In addition to (1.4) and (1.5), Algorithm FP-SQP requires the perturbed step $\widetilde{\Delta z}^{k}$ to satisfy

$$
d_{i}\left(z^{k}+\widetilde{\Delta z}{ }^{k}\right)=d_{i}\left(z^{k}\right)+\nabla d_{i}\left(z^{k}\right)^{T} \Delta z^{k}, \quad \forall i \in \mathcal{W}_{k}
$$

and

$$
\left\|\Delta z^{k}-\widetilde{\Delta z}\right\|=O\left(\left\|\Delta z^{k}\right\|^{2}\right) .
$$

We make the following notes about Assumption 5

- For iterations $k$ at which a step if taken (the "successful" iterations), we have that $\widetilde{\Delta z}^{k}=z^{k+1}-z^{k}$, which approaches zero by Assumption 5(a). Hence, by (1.6), and defining $\mathcal{K}$ to be the subsequence of successful iterations, we have that

$$
\lim _{k \in \mathcal{K}}\left\|\Delta z^{k}\right\|=\lim _{k \in \mathcal{K}}\left\|\widetilde{\Delta z}^{k}\right\|=0
$$


- The condition (4.2) is an explicit form of "second-order correction," a family of techniques that are often needed to ensure fast local convergence of SQP algorithms.

- It follows from (1.6) and (4.3) that

$$
\left\|\Delta z^{k}-\widetilde{\Delta z}^{k}\right\|=O\left(\left\|\widetilde{\Delta z}^{k}\right\|^{2}\right) .
$$

We start with a technical result to show that the various requirements on the perturbed step $\widetilde{\Delta z}^{k}$ are consistent. Note that this result is merely an existence result. It is not intended to show a practical way of obtaining $\widetilde{\Delta z}^{k}$. There may be other (less expensive, problem-dependent) ways to calculate the perturbed step that result in satisfaction of all the required conditions.

Lemma 4.1. Suppose that Assumption 4 and Assumptions 5(a),(b) hold. Then for all sufficiently large $k$, it is possible to choose the trust-region radius $\Delta_{k}$ small enough that there exists $\widetilde{\Delta z}^{k}$ satisfying (1.4), (1.5), (4.2), and (4.3).

Proof. Assume first that $k$ is chosen large enough that $\mathcal{W}_{k}=\mathcal{A}^{*}$. We prove the result constructively, generating $\widetilde{\Delta z}^{k}$ as the solution of the following problem:

$$
\begin{aligned}
& \min _{w} \frac{1}{2}\left\|w-\Delta z^{k}\right\|_{2}^{2} \text { s.t. } \\
c\left(z^{k}+w\right)= & 0, \\
d_{i}\left(z^{k}+w\right)= & d_{i}\left(z^{k}\right)+\nabla d_{i}\left(z^{k}\right)^{T} \Delta z^{k}, \quad \forall i \in \mathcal{W}_{k} .
\end{aligned}
$$

When the right hand sides of $(4.6 \mathrm{~b}),(4.6 \mathrm{c})$ are replaced by $c\left(z^{k}+\Delta z^{k}\right)$ and $d_{i}\left(z^{k}+\right.$ $\left.\Delta z^{k}\right)$, respectively, the solution is $w=\Delta z^{k}$. By the smoothness assumptions on $c$ and $d$, these modified right-hand sides represent only an $O\left(\left\|\Delta z^{k}\right\|^{2}\right)$ perturbation of the right-hand sides in (4.6b), (4.6c). Note that the Jacobian of the constraints (4.6b), (4.6c) has full row rank at $z^{k}+\Delta z^{k}$, because of Assumption 4(b) and Assumption 5(a). Hence, the Jacobian matrix of the KKT conditions for the problem (4.6) (which is a "square" system of nonlinear equations) is nonsingular at $z^{k}+\Delta z^{k}$, and a straightforward application of the implicit function theorem to this system yields that the solution $w=\widetilde{\Delta z}^{k}$ of (4.6) satisfies the property (4.3) for all $k$ sufficiently large. The condition (4.2) is an immediate consequence of (4.6c).

By decreasing $\Delta_{k}$ if necessary and using $\left\|\Delta z_{k}\right\| \leq \delta \Delta_{k}$, we can derive (1.5) as a consequence of (4.3).

Because of $(1.2 \mathrm{~b})$, we have

$$
d_{i}\left(z^{k}+\widetilde{\Delta z}{ }^{k}\right)=d_{i}\left(z^{k}\right)+\nabla d_{i}\left(z^{k}\right)^{T} \Delta z^{k} \leq 0, \quad \forall i \in \mathcal{A}^{*},
$$

while for $i \notin \mathcal{A}^{*}$ we have from $d_{i}\left(z^{*}\right)<0$ and Assumption 5(a) that

$$
d_{i}\left(z^{k}+\widetilde{\Delta z}^{k}\right)=d_{i}\left(z^{k}\right)+O\left(\Delta_{k}\right) \leq(1 / 2) d_{i}\left(z^{*}\right)<0
$$

for all $k$ sufficiently large and $\Delta_{k}$ sufficiently small. For the equality constraints we have immediately from (4.6b) that $c\left(z^{k}+\widetilde{\Delta z}^{k}\right)=0$. Hence $z^{k}+\widetilde{\Delta z}^{k} \in \mathcal{F}$, so condition (1.4) is also satisfied.

We assume that the Hessian matrix $H_{k}$ in the subproblem (1.2), (1.3) at $z=z^{k}$ is the Hessian of the Lagrangian $\mathcal{L}$ evaluated at this point, with appropriate estimates 
of the multipliers $\mu^{k}$ and $\lambda^{k}$; that is,

$$
H_{k}=\nabla_{z z}^{2} \mathcal{L}\left(z^{k}, \mu^{k}, \lambda^{k}\right)=\nabla^{2} f\left(z^{k}\right)+\sum_{i=1}^{m} \mu_{i}^{k} \nabla^{2} c_{i}\left(z^{k}\right)+\sum_{i=1}^{r} \lambda_{i}^{k} \nabla^{2} d_{i}\left(z^{k}\right) .
$$

We show now that with this choice of $H_{k}$, the ratio $\rho_{k}$ of actual to predicted decrease is close to 1 when $k$ is sufficiently large and the steps $\Delta z^{k}$ and $\widetilde{\Delta z}^{k}$ are sufficiently small. We prove the result specifically for the Euclidean-norm trust region; a minor generalization yields the proof for general $p \in[1, \infty]$.

Lemma 4.2. Suppose that $p=2$ in (1.3), that Assumptions 1, 4, and 5 hold, and that $H_{k}$ is defined by (4.7). Then there is a threshold value $\Delta_{\tau}$ and an index $K_{1}$ such that if $k \geq K_{1}$ and $\left\|D_{k} \Delta z^{k}\right\|_{2} \leq \Delta_{\tau}$, we have $\rho_{k} \geq 1 / 2$, where $\rho_{k}$ is defined by (2.1).

Proof. Note first that we can use $\Delta_{\tau}$ to control the size of both $\Delta z^{k}$ and $\widetilde{\Delta z}^{k}$, since from Assumption 1 we have $\left\|\Delta z^{k}\right\| \leq \delta\left\|D_{k} \Delta z^{k}\right\|_{2} \leq \delta \Delta_{\tau}$, while from (1.6) we have $\left\|\widetilde{\Delta z}{ }^{k}\right\| \leq(3 / 2)\left\|\Delta z^{k}\right\|$.

From (2.1) we have

$$
\rho_{k}=1+\frac{f\left(z^{k}\right)-f\left(z^{k}+\widetilde{\Delta z}^{k}\right)+m_{k}\left(\Delta z^{k}\right)}{-m_{k}\left(\Delta z^{k}\right)} .
$$

we prove the result by showing that the numerator of the final term in this expression is $o\left(\|\widetilde{\Delta z}\|^{k}\right)$, while the denominator is $\Omega\left(\left\|\Delta z^{k}\right\|^{2}\right)$.

We assume initially that $K_{1}$ is large enough that $\mathcal{W}_{k}=\mathcal{A}^{*}$ for all $k \geq K_{1}$. We work first with the numerator in (4.8). By elementary manipulation, using Taylor's theorem and the definition of $m_{k}(\cdot)$, we have for some $\theta^{f} \in(0,1)$ that

$$
\begin{aligned}
& f\left(z^{k}\right)-f\left(z^{k}+\widetilde{\Delta z}^{k}\right)+m_{k}\left(\Delta z^{k}\right) \\
& =-\nabla f\left(z^{k}\right)^{T} \widetilde{\Delta z}^{k}-\frac{1}{2}\left(\widetilde{\Delta z}^{k}\right)^{T} \nabla^{2} f\left(z^{k}+\theta^{f} \widetilde{\Delta z}^{k}\right) \widetilde{\Delta z}^{k}+\nabla f\left(z^{k}\right)^{T} \Delta z^{k}+\frac{1}{2}\left(\Delta z^{k}\right)^{T} H_{k} \Delta z^{k} \\
& =\left(\nabla f\left(z^{k}\right)+H_{k} \widetilde{\Delta z}^{k}\right)^{T}\left(\Delta z^{k}-\widetilde{\Delta z}^{k}\right)+\frac{1}{2}\left(\widetilde{\Delta z}^{k}\right)^{T}\left(H_{k}-\nabla^{2} f\left(z^{k}+\theta^{f} \widetilde{\Delta z}^{k}\right)\right) \widetilde{\Delta z}^{k} \\
& \quad \quad+O\left(\left\|\Delta z^{k}-\widetilde{\Delta z}^{k}\right\|^{2}\right) \\
& =\nabla f\left(z^{k}\right)^{T}\left(\Delta z^{k}-\widetilde{\Delta z}^{k}\right)+\frac{1}{2}\left(\widetilde{\Delta z}^{k}\right)^{T}\left(H_{k}-\nabla^{2} f\left(z^{k}\right)\right) \widetilde{\Delta z}^{k}+o\left(\left\|\widetilde{\Delta z}^{k}\right\|^{2}\right)
\end{aligned}
$$

where we used (4.5), boundedness of $H_{k}$, and continuity of $\nabla^{2} f$ to derive the final equality. Now from (1.2b) and continuity of $\nabla^{2} c_{i}$ for all $i=1,2, \ldots, m$ (Assumption 4(a)), we have

$$
\begin{aligned}
& 0=c_{i}\left(z^{k}+\widetilde{\Delta z}^{k}\right) \\
& =c_{i}\left(z^{k}\right)+\nabla c_{i}\left(z^{k}\right)^{T} \widetilde{\Delta z}^{k}+\frac{1}{2}\left(\widetilde{\Delta z}^{k}\right)^{T} \nabla^{2} c_{i}\left(z^{k}\right) \widetilde{\Delta z}^{k}+o\left(\left\|\widetilde{\Delta z}^{k}\right\|^{2}\right) \\
& =\nabla c_{i}\left(z^{k}\right)^{T}\left(\widetilde{\Delta z}-\Delta z^{k}\right)+\frac{1}{2}\left(\widetilde{\Delta z}^{k}\right)^{T} \nabla^{2} c_{i}\left(z^{k}\right) \widetilde{\Delta z}^{k}+o\left(\left\|\widetilde{\Delta z}^{k}\right\|^{2}\right) .
\end{aligned}
$$

From (4.2), we have for all $i \in \mathcal{A}^{*}$ that

$$
\begin{aligned}
0 & =d_{i}\left(z^{k}+\widetilde{\Delta z}^{k}\right)-d_{i}\left(z^{k}\right)-\nabla d_{i}\left(z^{k}\right)^{T} \Delta z^{k} \\
& =\nabla d_{i}\left(z^{k}\right)^{T}\left(\widetilde{\Delta z}^{k}-\Delta z^{k}\right)+\frac{1}{2}\left(\widetilde{\Delta z}^{k}\right)^{T} \nabla^{2} d_{i}\left(z^{k}\right) \widetilde{\Delta z}+o\left(\|\widetilde{\Delta z}\|^{k}\right) .
\end{aligned}
$$


For $i \notin \mathcal{A}^{*}$, we have from $\lambda_{i}^{k} \rightarrow \lambda_{i}^{*}=0$ and (4.5) that

$$
\lambda_{i}^{k} \nabla d_{i}\left(z^{k}\right)^{T}\left(\widetilde{\Delta z}-\Delta z^{k}\right)+\frac{1}{2} \lambda_{i}^{k}\left(\widetilde{\Delta z}^{k}\right)^{T} \nabla^{2} d_{i}\left(z^{k}\right) \widetilde{\Delta z}{ }^{k}=o\left(\|\widetilde{\Delta z}\|^{2}\right) .
$$

We now multiply equations (4.10) and (4.11) by their corresponding Lagrange multipliers ( $\mu_{i}^{k}$ and $\lambda_{i}^{k}$, respectively), and subtract them together with (4.12) from (4.9), to obtain

$$
\begin{aligned}
& f\left(z^{k}\right)-f\left(z^{k}+\widetilde{\Delta z}{ }^{k}\right)+m_{k}\left(\Delta z^{k}\right) \\
& =\left(\nabla f\left(z^{k}\right)+\nabla c\left(z^{k}\right) \mu^{k}+\nabla d\left(z^{k}\right) \lambda^{k}\right)^{T}\left(\Delta z^{k}-\widetilde{\Delta z}^{k}\right) \\
& +\frac{1}{2}\left(\widetilde{\Delta z}^{k}\right)^{T}\left[H_{k}-\nabla^{2} f\left(z^{k}\right)-\sum_{i=1}^{m} \mu_{i}^{k} \nabla^{2} c_{i}\left(z^{k}\right)-\sum_{i=1}^{r} \lambda_{i}^{k} \nabla^{2} d_{i}\left(z^{k}\right)\right] \widetilde{\Delta z}^{k} \\
& +o\left(\|\widetilde{\Delta z}\|^{2}\right) \\
& =O\left(\left\|\left(z^{k}, \mu^{k}, \lambda^{k}\right)-\left(z^{*}, \mu^{*}, \lambda^{*}\right)\right\|\right)\left\|\Delta z^{k}-\widetilde{\Delta z}^{k}\right\|+o\left(\left\|\widetilde{\Delta z}^{k}\right\|^{2}\right) \\
& =o\left(\left\|\widetilde{\Delta z}^{k}\right\|^{2}\right)
\end{aligned}
$$

where we used the KKT condition (1.8a) at $(z, \mu, \lambda)=\left(z^{*}, \mu^{*}, \lambda^{*}\right)$ and the definition (4.7) to derive the second equality, and Assumption 5(a),(c) together with (4.5) to derive the third equality. Hence we have shown that the numerator of the last term in (4.8) is $o\left(\left\|\widetilde{\Delta z}^{k}\right\|^{2}\right)$.

In the remainder of the proof we use the following shorthand notation for the Hessian of the Lagrangian:

$$
\left(\nabla_{z z}^{2} \mathcal{L}\right)_{k}=\nabla_{z z}^{2} \mathcal{L}\left(z^{k}, \mu^{k}, \lambda^{k}\right) ; \quad\left(\nabla_{z z}^{2} \mathcal{L}\right)_{*}=\nabla_{z z}^{2} \mathcal{L}\left(z^{*}, \mu^{*}, \lambda^{*}\right) .
$$

Given $p=2$ in (1.3), we see that the KKT conditions for $\Delta z^{k}$ to be a solution of (1.2), (1.3) at $z=z^{k}$ are that there exist Lagrange multipliers $\bar{\mu}^{k}, \bar{\lambda}^{k}$, and $\gamma_{k}$ such that

$$
\begin{aligned}
\nabla f\left(z^{k}\right)+\left(\nabla_{z z}^{2} \mathcal{L}\right)_{k} \Delta z^{k}+\nabla c\left(z^{k}\right) \bar{\mu}^{k}+\nabla d\left(z^{k}\right) \bar{\lambda}^{k}+\gamma_{k} D_{k}^{T} D_{k} \Delta z^{k} & =0 \\
c\left(z^{k}\right)+\nabla c\left(z^{k}\right)^{T} \Delta z^{k} & =0 \\
0 \geq d\left(z^{k}\right)+\nabla d\left(z^{k}\right)^{T} \Delta z^{k} \perp \bar{\lambda}^{k} & \geq 0 \\
0 \geq\left\|D_{k} \Delta z^{k}\right\|_{2}^{2}-\Delta_{k}^{2} \perp \gamma_{k} & \geq 0
\end{aligned}
$$

where $\gamma_{k}$ is the Lagrange multiplier for the trust-region constraint $\left\|D_{k} \Delta z^{k}\right\|_{2}^{2} \leq \Delta_{k}^{2}$. From (4.15b), (4.15c), and feasibility of $z^{k}$, we have

$$
\begin{aligned}
& \left(\bar{\mu}^{k}\right)^{T} \nabla c\left(z^{k}\right)^{T} \Delta z^{k}=-\left(\bar{\mu}^{k}\right)^{T} c\left(z^{k}\right)=0 \\
& \left(\bar{\lambda}^{k}\right)^{T} \nabla d\left(z^{k}\right)^{T} \Delta z^{k}=-\left(\bar{\lambda}^{k}\right)^{T} d\left(z^{k}\right) \geq 0 .
\end{aligned}
$$

We turn now to the denominator in (4.8), and show that it has size $\Omega\left(\left\|\Delta z^{k}\right\|^{2}\right)$ for all $k$ sufficiently large. From the definition of $m_{k}(\cdot),(4.7)$, and (4.14a), we have

$$
\begin{aligned}
-m_{k}\left(\Delta z^{k}\right) & =-\nabla f\left(z^{k}\right)^{T} \Delta z^{k}-\frac{1}{2}\left(\Delta z^{k}\right)^{T}\left(\nabla_{z z}^{2} \mathcal{L}\right)_{k} \Delta z^{k} \\
& =-\left(\Delta z^{k}\right)^{T}\left(\nabla f\left(z^{k}\right)+\left(\nabla_{z z}^{2} \mathcal{L}\right)_{k} \Delta z^{k}\right)+\frac{1}{2}\left(\Delta z^{k}\right)^{T}\left(\nabla_{z z}^{2} \mathcal{L}\right)_{k} \Delta z^{k} .
\end{aligned}
$$


By substituting from (4.15a), then using (4.15b) and (4.16), we obtain

$$
\begin{aligned}
-m_{k}\left(\Delta z^{k}\right)= & \left(\Delta z^{k}\right)^{T}\left(\nabla c\left(z^{k}\right) \bar{\mu}^{k}+\nabla d\left(z^{k}\right) \bar{\lambda}^{k}+\gamma_{k} D_{k}^{T} D_{k} \Delta z^{k}\right) \\
& +\frac{1}{2}\left(\Delta z^{k}\right)^{T}\left(\nabla_{z z}^{2} \mathcal{L}\right)_{k} \Delta z^{k} \\
= & -d\left(z^{k}\right)^{T} \bar{\lambda}^{k}+\gamma_{k}\left\|D_{k} \Delta z^{k}\right\|_{2}^{2}+\frac{1}{2}\left(\Delta z^{k}\right)^{T}\left(\nabla_{z z}^{2} \mathcal{L}\right)_{k} \Delta z^{k} .
\end{aligned}
$$

By using Assumption 1, we obtain

$$
-m_{k}\left(\Delta z^{k}\right) \geq-d\left(z^{k}\right)^{T} \bar{\lambda}^{k}+\gamma_{k} \delta^{-2}\left\|\Delta z^{k}\right\|_{2}^{2}+\frac{1}{2}\left(\Delta z^{k}\right)^{T}\left(\nabla_{z z}^{2} \mathcal{L}\right)_{k} \Delta z^{k} .
$$

We now define the constant $\bar{\gamma}$ as follows:

$$
\bar{\gamma} \stackrel{\text { def }}{=} \max \left(2 \delta^{2}\left\|\left(\nabla_{z z}^{2} \mathcal{L}\right)_{*}\right\|_{2}, 1\right) .
$$

By increasing $K_{1}$ if necessary, we have by smoothness of $\mathcal{L}$ together with Assumption $5(\mathrm{a}),(\mathrm{c})$ that

$$
\left\|\left(\nabla_{z z}^{2} \mathcal{L}\right)_{k}\right\|_{2} \leq 2\left\|\left(\nabla_{z z}^{2} \mathcal{L}\right)_{*}\right\|_{2} \leq \delta^{-2} \bar{\gamma}, \quad \forall k \geq K_{1} .
$$

We derive the estimate for $-m_{k}\left(\Delta z^{k}\right)$ from (4.17) by considering two cases. In the first case, we assume that $\gamma_{k} \geq \bar{\gamma}$. We then have from (4.17), using (4.16b), that the following bound holds for all $k \geq K_{1}$ :

$$
\begin{aligned}
-m_{k}\left(\Delta z^{k}\right) & \geq \gamma_{k} \delta^{-2}\left\|\Delta z^{k}\right\|_{2}^{2}+\frac{1}{2}\left(\Delta z^{k}\right)^{T}\left(\nabla_{z z}^{2} \mathcal{L}\right)_{k} \Delta z^{k} \\
& \geq \bar{\gamma} \delta^{-2}\left\|\Delta z^{k}\right\|_{2}^{2}-\frac{1}{2}\left\|\Delta z^{k}\right\|^{2}\left\|\left(\nabla_{z z}^{2} \mathcal{L}\right)_{k}\right\|_{2} \\
& \geq \frac{1}{2} \bar{\gamma} \delta^{-2}\left\|\Delta z^{k}\right\|_{2}^{2}
\end{aligned}
$$

so we see that the estimate $-m_{k}\left(\Delta z^{k}\right)=\Omega\left(\left\|\Delta z^{k}\right\|^{2}\right)$ is satisfied in this case .

In the second case of $\gamma_{k} \leq \bar{\gamma}$, a little more analysis is needed. We show first that

$$
\lim _{k \rightarrow \infty, \gamma_{k} \leq \bar{\gamma}}\left(\bar{\mu}^{k}, \bar{\lambda}^{k}\right)=\left(\mu^{*}, \lambda^{*}\right) .
$$

By choosing $\Delta_{\tau}$ small enough and increasing $K_{1}$ if necessary, we have when $\left\|D_{k} \Delta z^{k}\right\| \leq \Delta_{\tau}$ and $k \geq K_{1}$ that

$$
\begin{aligned}
& i \notin \mathcal{W}_{k}=\mathcal{A}^{*} \\
& \Rightarrow d_{i}\left(z^{k}\right)+\nabla d_{i}\left(z^{k}\right)^{T} \Delta z^{k}=d_{i}\left(z^{*}\right)+O\left(\left\|z^{k}-z^{*}\right\|\right)+O\left(\left\|\Delta z^{k}\right\|\right) \leq(1 / 2) d_{i}\left(z^{*}\right)<0,
\end{aligned}
$$

where we used Assumption 5(a) for the first equality. Hence, from (4.15c), we have $\bar{\lambda}_{i}^{k}=0$ for all $i \notin \mathcal{A}^{*}$. By rearranging (4.15a), we therefore have

$$
\nabla c\left(z^{k}\right) \bar{\mu}^{k}+\nabla d_{\mathcal{A}^{*}}\left(z^{k}\right) \bar{\lambda}_{\mathcal{A}^{*}}^{k}=-\nabla f\left(z^{k}\right)-\left(\nabla_{z z}^{2} \mathcal{L}\right)_{k} \Delta z^{k}-\gamma_{k} D_{k}^{T} D_{k} \Delta z^{k} .
$$

By comparing this expression with the KKT condition for $z^{*}$, namely,

$$
\nabla c\left(z^{*}\right) \mu^{*}+\nabla d_{\mathcal{A}^{*}}\left(z^{*}\right) \lambda_{\mathcal{A}^{*}}^{*}=-\nabla f\left(z^{*}\right),
$$

and using the LICQ (Assumption 4(b)), Assumption 1, and $\gamma_{k} \leq \bar{\gamma}$, we obtain

$$
\left\|\left(\bar{\mu}^{k}, \bar{\lambda}_{\mathcal{A}^{*}}^{k}\right)-\left(\mu^{*}, \lambda_{\mathcal{A}^{*}}^{*}\right)\right\|=O\left(\left\|z^{k}-z^{*}\right\|\right)+O\left(\left\|\Delta z^{k}\right\|\right) \rightarrow 0 .
$$


Hence, by strict complementarity (Assumption 4(c)), and by increasing $K_{1}$ again if necessary, we can identify a constant $\bar{\lambda}_{\text {min }}>0$ such that

$$
\bar{\lambda}_{i}^{k} \geq \bar{\lambda}_{\min }, \forall i \in \mathcal{A}^{*}, \quad \forall k \geq K_{1} \text { with } \gamma_{k} \leq \bar{\gamma} .
$$

Therefore, by the complementarity condition (4.15c), we have that

$$
\nabla d_{\mathcal{A}^{*}}\left(z^{k}\right)^{T} \Delta z^{k}=-d_{\mathcal{A}^{*}}\left(z^{k}\right) .
$$

Using this expression together with (4.15b), we deduce that

$$
\begin{aligned}
{\left[\begin{array}{c}
\nabla c\left(z^{*}\right)^{T} \\
\nabla d_{\mathcal{A}^{*}}\left(z^{*}\right)^{T}
\end{array}\right] \Delta z^{k} } & =\left[\begin{array}{c}
\left(\nabla c\left(z^{*}\right)-\nabla c\left(z^{k}\right)\right)^{T} \Delta z^{k} \\
-d_{\mathcal{A}^{*}}\left(z^{k}\right)+\left(\nabla d_{\mathcal{A}^{*}}\left(z^{*}\right)-\nabla d_{\mathcal{A}^{*}}\left(z^{k}\right)\right)^{T} \Delta z^{k}
\end{array}\right] \\
& =O\left(\left\|d_{\mathcal{A}^{*}}\left(z^{k}\right)\right\|\right)+O\left(\left\|z^{k}-z^{*}\right\|\left\|\Delta z^{k}\right\|\right) .
\end{aligned}
$$

By full row rank of the coefficient matrix on the left-hand side of (4.22), we have that there exists a vector $s^{k}$ with

$$
\begin{aligned}
{\left[\begin{array}{c}
\nabla c\left(z^{*}\right)^{T} \\
\nabla d_{\mathcal{A}^{*}}\left(z^{*}\right)^{T}
\end{array}\right] s^{k} } & =\left[\begin{array}{c}
\nabla c\left(z^{*}\right)^{T} \\
\nabla d_{\mathcal{A}^{*}}\left(z^{*}\right)^{T}
\end{array}\right] \Delta z^{k} \\
\left\|s^{k}\right\| & =O\left(\left\|d_{\mathcal{A}^{*}}\left(z^{k}\right)\right\|\right)+O\left(\left\|z^{k}-z^{*}\right\|\left\|\Delta z^{k}\right\|\right) .
\end{aligned}
$$

Since the vector $\Delta z^{k}-s^{k}$ satisfies the conditions on $v$ in the second-order sufficient conditions (Assumptions 4(d)), we have

$$
\left(\Delta z^{k}-s^{k}\right)^{T}\left(\nabla_{z z}^{2} \mathcal{L}\right)_{*}\left(\Delta z^{k}-s^{k}\right) \geq \alpha\left\|\Delta z^{k}-s^{k}\right\|_{2}^{2},
$$

so that by increasing $K_{1}$ again if necessary, we have by Assumption 5(a),(c) that

$$
\left(\Delta z^{k}-s^{k}\right)^{T}\left(\nabla_{z z}^{2} \mathcal{L}\right)_{k}\left(\Delta z^{k}-s^{k}\right) \geq \frac{1}{2} \alpha\left\|\Delta z^{k}-s^{k}\right\|_{2}^{2}, \quad \forall k \geq K_{1} .
$$

By using this inequality together with (4.23b) and Assumption 5(a), we obtain (again increasing $K_{1}$ if needed) that

$$
\begin{aligned}
& \left(\Delta z^{k}\right)^{T}\left(\nabla_{z z}^{2} \mathcal{L}\right)_{k} \Delta z^{k} \\
& =\left(\Delta z^{k}-s^{k}\right)^{T}\left(\nabla_{z z}^{2} \mathcal{L}\right)_{k}\left(\Delta z^{k}-s^{k}\right)+O\left(\left\|s^{k}\right\|\left\|\Delta z^{k}\right\|\right)+O\left(\left\|s^{k}\right\|^{2}\right) \\
& \geq \frac{1}{2} \alpha\left\|\Delta z^{k}-s^{k}\right\|_{2}^{2}+O\left(\left\|\Delta z^{k}\right\|\left\|s^{k}\right\|\right)+O\left(\left\|s^{k}\right\|^{2}\right) \\
& =\frac{1}{2} \alpha\left\|\Delta z^{k}\right\|_{2}^{2}+O\left(\left\|\Delta z^{k}\right\|\left\|s^{k}\right\|\right)+O\left(\left\|s^{k}\right\|^{2}\right) \\
& =\frac{1}{2} \alpha\left\|\Delta z^{k}\right\|_{2}^{2}+O\left(\left\|d_{\mathcal{A}^{*}}\left(z^{k}\right)\right\|\left\|\Delta z^{k}\right\|\right)+O\left(\left\|d_{\mathcal{A}^{*}}\left(z^{k}\right)\right\|^{2}\right) \\
& \quad \quad+O\left(\left\|d_{\mathcal{A}^{*}}\left(z^{k}\right)\right\|\left\|z^{k}-z^{*}\right\|\left\|\Delta z^{k}\right\|\right)+o\left(\left\|\Delta z^{k}\right\|^{2}\right) \\
& \geq \frac{1}{4} \alpha\left\|\Delta z^{k}\right\|_{2}^{2}+O\left(\left\|d_{\mathcal{A}^{*}}\left(z^{k}\right)\right\|\left\|\Delta z^{k}\right\|\right)+O\left(\left\|d_{\mathcal{A}^{*}}\left(z^{k}\right)\right\|^{2}\right)
\end{aligned}
$$

for all $k \geq K_{1}$ with $\gamma_{k} \leq \bar{\gamma}$. Because of (4.21), and since $\bar{\lambda}_{i}^{k}=0$ for $i \notin \mathcal{A}^{*}$, we have

$$
-\left(\bar{\lambda}^{k}\right)^{T} d\left(z^{k}\right)=\sum_{i \in \mathcal{A}^{*}} \bar{\lambda}_{i}^{k}\left(-d_{i}\left(z^{k}\right)\right) \geq \bar{\lambda}_{\min }\left\|d_{\mathcal{A}^{*}}\left(z^{k}\right)\right\|_{1} .
$$

By substituting (4.24) and (4.25) into (4.17), and dropping the second term on the right-hand side of (4.17) (which is positive in any case), we obtain

$$
\begin{aligned}
-m_{k}\left(\Delta z^{k}\right) & \geq-d\left(z^{k}\right)^{T} \bar{\lambda}^{k}+\frac{1}{2}\left(\Delta z^{k}\right)^{T}\left(\nabla_{z z}^{2} \mathcal{L}\right)_{k} \Delta z^{k} \\
& \geq \bar{\lambda}_{\min }\left\|d_{\mathcal{A}^{*}}\left(z^{k}\right)\right\|_{1}+(1 / 8) \alpha\left\|\Delta z^{k}\right\|_{2}^{2}+O\left(\left\|d_{\mathcal{A}^{*}}\left(z^{k}\right)\right\|\left\|\Delta z^{k}\right\|\right)+O\left(\left\|d_{\mathcal{A}^{*}}\left(z^{k}\right)\right\|^{2}\right) \\
& \geq(1 / 8) \alpha\left\|\Delta z^{k}\right\|_{2}^{2}, \quad \forall k \geq K_{1} .
\end{aligned}
$$


The last inequality holds because the term $\bar{\lambda}_{\text {min }}\left\|d_{\mathcal{A}^{*}}\left(z^{k}\right)\right\|_{1}$ dominates the remainder terms (after possibly another decrease of $\Delta_{\tau}$ and increase of $K_{1}$ ).

We conclude from (4.20) and (4.26) that for all $k$ sufficiently large, we have $-m_{k}\left(\Delta z^{k}\right)=\Omega\left(\left\|\Delta z^{k}\right\|^{2}\right)$. By combining this estimate with (4.13) and (4.8), and using (1.6), we obtain that

$$
\rho_{k}=1+\frac{o\left(\|\widetilde{\Delta z}\|^{2}\right)}{\Omega\left(\left\|\Delta z^{k}\right\|^{2}\right)}=1+\frac{o\left(\left\|\Delta z^{k}\right\|^{2}\right)}{\Omega\left(\left\|\Delta z^{k}\right\|^{2}\right)} .
$$

Hence by decreasing $\Delta_{\tau}$ further if necessary, we have $\rho_{k}>1 / 2$ whenever $k \geq K_{1}$ and $\left\|D_{k} \Delta z^{k}\right\| \leq \Delta_{\tau}$, as claimed.

The next lemma takes a few more steps toward our superlinear convergence result.

Lemma 4.3. Suppose that $p=2$ in (1.3), that Assumptions 1, 4, and 5 hold, and that $H_{k}$ is defined by (4.7). Let $K_{1}$ and $\Delta_{\tau}$ be as defined in Lemma 4.2. Then the following are true:

(a) For all $k \geq K_{1}$, we have $\Delta_{k} \geq \min \left(\Delta_{K_{1}}, \Delta_{\tau} / 2\right)$.

(b) There is an index $K_{2}$ such that the trust-region bound (1.3) is inactive at all successful iterations $k$ with $k \geq K_{2}$.

Proof. For (a), Lemma 4.2 indicates that for $k \geq K_{1}$, the trust-region radius can be decreased only when $\left\|D_{k} \Delta z^{k}\right\|_{2}>\Delta_{\tau}$. Since Algorithm FP-SQP decreases the trust region by setting it to $(1 / 2)\left\|D_{k} \Delta z^{k}\right\|_{2}$, we must have $\Delta_{k+1} \geq \Delta_{\tau} / 2$ after any such decrease. On the other hand, if no decreases occur after iteration $K_{1}$, we have $\Delta_{k} \geq \Delta_{K_{1}}$ for all $k \geq K_{1}$. The claim follows by combining these two observations.

For (b), we observed in (4.4) that $\left\|\Delta z^{k}\right\| \rightarrow 0$ for the successful steps, while from part (a), the trust-region radius is bounded below by a positive quantity. Hence, we can identify an index $K_{2}$ with the required property.

TheOrem 4.4. Suppose that $p=2$ in (1.3), that Assumptions 1, 4, and 5 hold, and that $H_{k}$ is defined by (4.7). Then the sequence $\left\{z^{k}\right\}$ converges Q-superlinearly to $z^{*}$.

Proof. At all successful iterations $k$ with $k \geq K_{2}$, the step $\Delta z^{k}$ is a (full) standard SQP step. Hence by the known local convergence properties of SQP with an exact Hessian, we have that

$$
\left\|z^{k}+\Delta z^{k}-z^{*}\right\| \leq \beta\left\|z^{k}-z^{*}\right\|\left[\left\|z^{k}-z^{*}\right\|+\left\|\left(\mu^{k}, \lambda^{k}\right)-\left(\mu^{*}, \lambda^{*}\right)\right\|\right]=o\left(\left\|z^{k}-z^{*}\right\|\right),
$$

where $\beta$ is a constant, and we have used Assumption 5(a) and (c) to obtain the final equality. It follows from this expression that

$$
\left\|\Delta z^{k}\right\|=O\left(\left\|z^{k}-z^{*}\right\|\right) .
$$

Using this estimate together with (4.3), we have

$$
\begin{aligned}
\left\|z^{k+1}-z^{*}\right\| & =\left\|z^{k}+\widetilde{\Delta z}-z^{*}\right\| \\
& \leq\left\|z^{k}+\Delta z^{k}-z^{*}\right\|+\left\|\Delta z^{k}-\widetilde{\Delta z}^{k}\right\| \\
& =o\left(\left\|z^{k}-z^{*}\right\|\right)+O\left(\left\|\Delta z^{k}\right\|^{2}\right)=o\left(\left\|z^{k}-z^{*}\right\|\right),
\end{aligned}
$$

showing that $Q$-superlinear behavior occurs at all successful steps with $k \geq K_{2}$.

We show now that there is an index $K_{3} \geq K_{2}$ such that all iterations $k \geq K_{3}$ are successful. If not, then there are infinitely many unsuccessful iterations and the trust-region radius is reduced (by at least a factor of 2 ) at each such iteration. Since by Lemma $4.3(\mathrm{~b})$, the trust region is inactive at the successful steps, the radius is not increased at these steps. Hence, we have $\Delta_{k} \downarrow 0$, which contradicts Lemma 4.3(a). 
5. Conclusions. We have described a simple feasibility perturbed trust-region SQP algorithm for nonlinear programming with good global and local convergence properties. As discussed above, we believe that the feasibility perturbation often can be carried out efficiently when the constraints are separable or otherwise structured. The companion report [15] describes application of the algorithm to optimal control problems with constraints on the inputs (controls).

We assert (without proof) the following result concerning global convergence to points satisfying second-order necessary conditions. When the assumptions used in Section 3 are satisfied, $z^{*}$ is a KKT limit point of the sequence $\left\{z^{k}\right\}$ at which LICQ and strict complementarity are satisfied, asymptotically exact estimates of $\left(\mu^{k}, \lambda^{k}\right)$ and $\mathcal{W}_{k}$ are available on the convergent subsequence $\mathcal{K}, H_{k}$ is chosen as in (4.7), and Assumption 5(d) is satisfied, then the following second-order necessary condition holds at $z^{*}$ :

$$
v^{T} \nabla_{z z}^{2} \mathcal{L}\left(z^{*}, \mu^{*}, \lambda^{*}\right) v \geq 0, \quad \forall v \text { s.t. } \nabla c\left(z^{*}\right)^{T} v=0, \quad \nabla d_{\mathcal{A}^{*}}\left(z^{*}\right)^{T} v=0,
$$

We omit the proof, which uses many of the same techniques as in Sections 3 and 4.

Acknowledgments. We thank Paul Tseng for suggesting Assumption 3. We are extremely grateful to the editor and to two anonymous referees whose careful reading and penetrating insights greatly improved the paper. We gratefully acknowledge the financial support of the industrial members of the Texas-Wisconsin Modeling and Control Consortium. In addition, research of the second author was supported in part by NSF grants \#CTS-0105360, while research of the first author was supported in part by NSF grants \#MTS-0086559, \#ACI-0196485, and \#EIA-0127857.

\section{Appendix A. Value Function of a Parametrized Linear Program.}

Here we prove Lemma 3.1. (3.1).

Proof. Note first that $C(z, 1) \geq 0$ for any feasible $z$, since $w=0$ is feasible for

We have $C(z, 1)=0$ if and only if $w=0$ is a solution of the problem (3.1). The bound $w^{T} w \leq 1$ is inactive at $w=0$, and the optimality conditions for (3.1) are then identical to the KKT conditions (1.8) for (1.1). Hence, $C(z, 1)=0$ if and only if $z$ satisfies the KKT conditions.

Suppose now that $\bar{z} \in \mathcal{F}$ satisfies MFCQ (1.10) but not the KKT conditions (1.8). Suppose for contradiction that there exists a sequence $\left\{z^{l}\right\}$ with $z^{l} \rightarrow \bar{z}, z^{l} \in \mathcal{F}$ such that

$$
0 \leq C\left(z^{l}, 1\right) \leq l^{-1}, \quad l=1,2,3, \ldots
$$

The KKT conditions for the solution $w^{l}$ of (3.1) at $z=z^{l}$ are that there exist multipliers $\mu^{l} \in \mathrm{R}^{m}, \lambda^{l} \in \mathrm{R}^{r}$, and $\beta^{l} \in \mathrm{R}$ such that:

$$
\begin{aligned}
\nabla f\left(z^{l}\right)+\nabla c\left(z^{l}\right) \mu^{l}+\nabla d\left(z^{l}\right) \lambda^{l}+2 \beta^{l} w^{l} & =0, \\
c\left(z^{l}\right)+\nabla c\left(z^{l}\right)^{T} w^{l} & =0, \\
d\left(z^{l}\right)+\nabla d\left(z^{l}\right)^{T} w^{l} \leq 0 & \perp \lambda^{l} \geq 0, \\
\left(w^{l}\right)^{T} w^{l}-1 \leq 0 & \perp \beta^{l} \geq 0 .
\end{aligned}
$$

We now verify that these are in fact optimality conditions for (3.1) by showing that MFCQ holds at $w^{l}$. We define the "linearized" active indices at $z^{l}$ as follows:

$$
\mathcal{A}_{l} \stackrel{\text { def }}{=}\left\{i=1,2, \ldots, r \mid d_{i}\left(z^{l}\right)+\nabla d_{i}\left(z^{l}\right)^{T} w^{l}=0\right\} .
$$


Since MFCQ holds for the original problem (1.1) at $\bar{z}$, we have by the logic in the proof of Lemma 2.1 that MFCQ is also satisfied at $z^{l}$ for all $l$ sufficiently large. Hence, there is a vector $v^{l}$ such that

$$
\nabla c\left(z^{l}\right)^{T} v^{l}=0 \text { and } \nabla d_{i}\left(z^{l}\right)^{T} v^{l}<0 \text { for all } i \in \mathcal{A}\left(z^{l}\right) .
$$

Consider now the vector

$$
u^{l}=-w^{l}+\epsilon v^{l}
$$

for some $\epsilon>0$ to be defined. We show that $u^{l}$ is an "MFCQ direction" for (3.1) at $w^{l}$, that is

$$
\begin{gathered}
\left\|w^{l}\right\|_{2}=1 \Rightarrow 2\left(w^{l}\right)^{T} u^{l}<0, \\
\nabla c\left(z^{l}\right)^{T} u^{l}=0, \\
i \in \mathcal{A}_{l} \cap \mathcal{A}\left(z^{l}\right) \Rightarrow \nabla d_{i}\left(z^{l}\right)^{T} u^{l}<0, \\
i \in \mathcal{A}_{l} \backslash \mathcal{A}\left(z^{l}\right) \Rightarrow \nabla d_{i}\left(z^{l}\right)^{T} u^{l}<0 .
\end{gathered}
$$

For (A.2a), we have when $\left\|w^{l}\right\|_{2}=1$ that

$$
\left(2 w^{l}\right)^{T} u^{l}=-2\left\|w^{l}\right\|_{2}^{2}+\epsilon\left(w^{l}\right)^{T} v^{l}=-2+\epsilon\left(w^{l}\right)^{T} v^{l}<0
$$

for all $\epsilon>0$ sufficiently small. The second condition (A.2b) obviously holds, since $\nabla c\left(z^{l}\right)^{T} w^{l}=0$ and $\nabla c\left(z^{l}\right)^{T} v^{l}=0$. For (A.2c), we have

$\nabla d_{i}\left(z^{l}\right)^{T} u^{l}=-\nabla d_{i}\left(z^{l}\right)^{T} w^{l}+\epsilon \nabla d_{i}\left(z^{l}\right)^{T} v^{l}=d_{i}\left(z^{l}\right)+\epsilon \nabla d_{i}\left(z^{l}\right)^{T} v^{l} \leq \epsilon \nabla d_{i}\left(z^{l}\right)^{T} v^{l}<0$,

for all $\epsilon>0$, where the second equality follows from $i \in \mathcal{A}_{l}$ and the third equality from $z^{l} \in \mathcal{F}$. For $(\mathrm{A} .2 \mathrm{~d})$, we have from $i \notin \mathcal{A}\left(z^{l}\right)$ that $d_{i}\left(z^{l}\right)<0$, and so

$$
\nabla d_{i}\left(z^{l}\right)^{T} u^{l}=-\nabla d_{i}\left(z^{l}\right)^{T} w^{l}+\epsilon \nabla d_{i}\left(z^{l}\right)^{T} v^{l}=d_{i}\left(z^{l}\right)+\epsilon \nabla d_{i}\left(z^{l}\right)^{T} v^{l}<0,
$$

for all $\epsilon>0$ sufficiently small. It is clearly possibly to choose $\epsilon$ in such a way that all the conditions (A.2) are satisfied, so we conclude that (A.1) are indeed optimality conditions for $w^{l}$.

From these relations, and using the fact that $z^{l} \in \mathcal{F}$, we have that

$$
\begin{aligned}
C\left(z^{l}, 1\right) & =-\nabla f\left(z^{l}\right)^{T} w^{l} \\
& =\left(w^{l}\right)^{T} \nabla c\left(z^{l}\right) \mu^{l}+\left(w^{l}\right)^{T} \nabla d\left(z^{l}\right) \lambda^{l}+2 \beta^{l}\left(w^{l}\right)^{T} w^{l} \\
& =-d\left(z^{l}\right)^{T} \lambda^{l}+2 \beta^{l} \geq 0
\end{aligned}
$$

By taking limits as $l \rightarrow \infty$, and since $-d\left(z^{l}\right)^{T} \lambda^{l}$ and $\beta^{l}$ are both nonnegative, we have from (A.3) that

$$
\beta^{l} \rightarrow 0, \quad d\left(z^{l}\right)^{T} \lambda^{l} \rightarrow 0
$$

Consider first the case in which there is a subsequence $\mathcal{K}$ of multipliers from (A.1), that is, $\left\{\mu^{l}, \lambda^{l}\right\}_{l \in \mathcal{K}}$ is bounded. By compactness, and taking a further subsequence of $\mathcal{K}$ if necessary, we can identify $\bar{\mu}$ and $\bar{\lambda} \geq 0$ such that

$$
\left(\mu^{l}, \lambda^{l}\right)_{l \in \mathcal{K}} \rightarrow(\bar{\mu}, \bar{\lambda}) .
$$


Then by taking limits in (A.1a), and using (A.4) and (1.7), we have that

$$
\nabla_{z} \mathcal{L}(\bar{z}, \bar{\mu}, \bar{\lambda})=0, \quad d(\bar{z})^{T} \bar{\lambda}=0 .
$$

By using these relations together with feasibility of $\bar{z}$, we see that $\bar{z}$ is a KKT point, which is a contradiction.

In the other case, the sequence $\left\{\mu^{l}, \lambda^{l}\right\}$ has no bounded subsequence. By taking another subsequence $\mathcal{K}$, we can identify a vector $(\hat{\mu}, \hat{\lambda})$ with $\|(\hat{\mu}, \hat{\lambda})\|_{2}=1$ and $\hat{\lambda} \geq 0$ such that

$$
\lim _{k \in \mathcal{K}} \frac{\left(\mu^{l}, \lambda^{l}\right)}{\left\|\left(\mu^{l}, \lambda^{l}\right)\right\|_{2}}=(\hat{\mu}, \hat{\lambda}), \quad \lim _{k \in \mathcal{K}}\left\|\left(\mu^{l}, \lambda^{l}\right)\right\|_{2}=\infty .
$$

By dividing both sides of (A.1a) by $\left\|\left(\mu^{l}, \lambda^{l}\right)\right\|_{2}$ and using (A.4), we obtain

$$
\nabla c(\bar{z}) \hat{\mu}+\nabla d(\bar{z}) \hat{\lambda}=0, \quad d(\bar{z})^{T} \hat{\lambda}=0, \quad \hat{\lambda} \geq 0 .
$$

It is easy to show that (A.7) together with the MFCQ (1.10) implies that $(\hat{\mu}, \hat{\lambda})=0$, which contradicts $\|(\hat{\mu}, \hat{\lambda})\|_{2}=1$ (see Clarke [4, pp. 235-236]).

Therefore, we obtain a contradiction, so that no sequence $\left\{z^{l}\right\}$ with the claimed properties exists, and therefore $C(z, 1)$ is bounded away from zero in a neighborhood of $\bar{z}$.

\section{REFERENCES}

[1] D. P. Bertsekas. Projected Newton methods for optimization problems with simple constraints. SIAM Journal on Control and Optimization, 20:221-246, 1982.

[2] J. R. Birge, L. Qi, and Z. Wei. A varaint of the Topkis-Veinott method for solving inequality constrained optimization problems. J. Appl. Math. Optim., 41:309-330, 2000.

[3] M.R. Celis, J.E. Dennis, and R.A. Tapia. A trust region strategy for nonlinear equality constrained optimization. In P.T. Boggs, R.H. Byrd, and R.B. Schnabel, editors, Numerical Optimization, pages 71-82. SIAM, 1985.

[4] F. H. Clarke. Optimization and Nonsmooth Analysis. John Wiley, New York, 1983.

[5] A. R. Conn, N. I. M. Gould, and Ph. L. Toint. Trust-Region Methods. MPS-SIAM Series on Optimization. SIAM, 2000.

[6] F. Facchinei, A. Fischer, and C. Kanzow. On the accurate identification of active constraints. SIAM Journal on Optimization, 9(1):14-32, 1998.

[7] R. Fletcher and E. Sainz de la Maza. Nonlinear programming and nonsmooth optimization by successive linear programming. Mathematical Programming, 43:235-256, 1989.

[8] M. Heinkenschloss. Projected sequential quadratic programming methods. SIAM Journal on Optimization, 6(2):373-417, 1996.

[9] A. J. Hoffman. On approximate solutions of systems of linear inequalities. Journal of Research of the National Bureau of Standards, 49:263-265, 1952.

[10] C. T. Lawrence and A. L. Tits. A computationally efficient feasible sequential quadratic programming algorithm. SIAM Journal on Optimization, 11(4):1092-1118, 2001.

[11] S. Lucidi, M. Sciandrone, and P. Tseng. Objective-derivative-free methods for constrained optimization. Mathematical Programming, Series A, 92:37-59, 2002.

[12] E. O. Omojokun. Trust-region algorithms for optimization with nonlinear equality and inequality constraints. PhD thesis, University of Colorado, Boulder, 1989.

[13] E. R. Panier and A. L. Tits. On combining feasibility, descent and superlinear convergence in inequality constrained optimization. Mathematical Programming, 59:261-276, 1993.

[14] S. M. Robinson. Stability theory for systems of inequalities, Part II: Differentiable nonlinear systems. SIAM Journal on Numerical Analysis, 13:497-513, 1976.

[15] M. J. Tenny, S. J. Wright, and J. B. Rawlings. Nonlinear model predictive control via feasibilityperturbed sequential quadratic programming. Optimization Technical Report 02-06, University of Wisconsin-Madison, Computer Sciences Department, August 2002. Also TexasWisconsin Modeling and Control Consortium Report TWMCC-2002-02. To appear in Computational Optimization and Applications. 
[16] S. J. Wright. Convergence of an inexact algorithm for composite nonsmooth optimization. IMA Journal of Numerical Analysis, 10:299-321, 1990.

[17] Y. Yuan. Conditions for convergence of a trust-region method for nonsmooth optimization. Mathematical Programming, 31:269-285, 1985. 